\title{
Expert Programmer versus Parallelizing Compiler: A Comparative Study of Two Approaches for Distributed Shared Memory
}

\author{
M. F. P. O'BOYLE AND J. M. BULL \\ Centre for Novel Computing, Department of Compuier Science, University of Manchester, Oxford Road, Manchester M13 9PL, UK
}

\begin{abstract}
This article critically examines current parallel programming practice and optimizing compiler development. The general strategies employed by compiler and programmer to optimize a Fortran program are described, and then illustrated for a specific case by applying them to a well-known scientific program, TRED2, using the KSR-1 as the target architecture. Extensive measurement is applied to the resulting versions of the program, which are compared with a version produced by a commercial optimizing compiler, KAP. The compiler strategy significantly outperforms KAP and does not fall far short of the performance achieved by the programmer. Following the experimental section each approach is critiqued by the other. Perceived flaws, advantages, and common ground are outlined, with an eye to improving both schemes. (c) 1996 by John Wiley \& Sons, Inc.
\end{abstract}

\section{INTRODUCTION}

Obtaining high performance from parallel computers is the goal of both programmers and optimizing compilers. Despite this obvious overlap in concern there has been little investigation into how each tries to achieve this goal, and whether either has anything to learn from the other. In this article we begin such an investigation by way of an experiment, where the experimental method, evaluation, and results are clearly defined. We also describe and compare the underlying mechanisms used by programmer and compiler writer in their attempt to parallelize a given program.

Received October 1994

Revised February 1995

(C) 1996 by John Wiley \& Sons, Inc.

Scientific Programming, Vol. 5, pp. 63-88 (1996)

CCC 1058-9244/96/010063-26
Historically, scientific programmers and compiler writers have worked in relative isolation for good reason; one is primarily concerned with reflecting a particular scientific model in a particular program while the other is concerned with implementing a language on a machine or family of machines. Both are concerned with preservation of meaning-one from model to program, the other from language to machine, but there is a difference in focus. Compiler writers are concerned with a range of general program constructs while programmers are more concerned with a single program (at a time) and its particular structure. Furthermore, programmers view a program in the light of the physical reality it is modeling, while compiler writers see it as a particular instance of all possible programs.

In parallel scientific computing, the emphasis on performance brings the perspectives of programmer and compiler writer closer together. On one hand there is a need for programmers to understand the features of a particular parallel ar- 
chitecture and of the compiler that tries to efficiently map their program to it. Conversely, compilers need to understand programs at a higher level of abstraction than just a syntax tree if they are going to perform well. In a sense the program is a contract or dialogue between programmer and compiler, where the former tries to explain to the latter what computation is to be done in a clear enough manner to enable efficient compilation to take place. Sometimes the need for clarity is so great that explicit language extensions are needed to tell the compiler not just what has to be done, but also how to do it.

The assumption that the programmer is the author of the program is by no means always true. Often a "dusty deck" is to be ported to a new machine, where the programmer may have to apply a process of reverse engineering to try to determine exactly what the program is trying to do before they reimplement it. This situation is due to a lack of integration between programmers and compiler writers. When the dusty deck was original written, it may well have been written in a high-level, easy-to-understand manner. However, because the compilers of the time could not deliver the required performance, the program was hand transformed into some obscure and contorted form so as to best utilize the machine. In effect, the programmer took on the role of the compiler for most of the optimization. Lnfortunately much of this transformation is architecture dependent and has to be undone before it can be mapped to a new machine. However, the contorted version is usually all that remains, so inevitably when a new architecture/compiler is used, it often gives miserable results when compared to the previous system, hindering progress in parallel computing.

In this situation the dialogue between programmer and compiler (i.e., the program) is no longer understandable to either in its present form and has to be rewritten before progress can be made. Precisely how this effort should be divided is the area of debate. In this article we try to address this question by examining the world views of both groups. We take a representative of each and ask them to describe their general methodology, and how they would implement a particular scientific program.

The chosen program, TRED2, is a 100-line Fortran program from the eigenvalue solver package Eispack. It reduces a symmetric matrix to symmetric tridiagonal form using accumulating orthogonal similarity transformations. The pro- gram contains imperfectly nested loops. IF statements, and GOTOs, and, as such, is typical of many programs found in scientific computing. It is long enough so as not to be trivial and extensively studied (such as matrix multiplication), but short enough to allow analysis of the resulting program behavior in a reasonable time.

A description of the machine used and its programming environment is given in Section 2. In Section 3 both the programmer and compiler writer* set out their approach to parallelization. Sections 4 and 5 describe the application of the two approaches to the implementation of TRED2 on the KSR-1. The performance of the resulting programs is reported in Section 6 and compared with the output of an existing commercial parallelizing compiler. The relation between the experiments can be seen in Figure 1. As the programmer and compiler writer are both authors of this article, there may be a tendency to overly agree, so. in Section 7 each approach is critiqued by the other. The advantages and disadvantages of the two approaches are outlined, and common ground between them identified, with an emphasis on how each scheme might be improved. We seek a more cooperative way forward for programmer and compiler, and outline further research in this area.

\section{THE KENDALL SQUARE RESEARCH KSR-1}

\subsection{Architecture}

The target machine for the experiment is the 32 cell Kendall Square Research KSR-1 computer installed at the Lniversity of Manchester. $t$ This is a distributed shared memory machine; it has a physically distributed memory but there is extensive hardware support for a single logical address space. Each cell consists of a $20-\mathrm{MHz}$ processor with a peak 64-bit floating point performance of $40 \mathrm{Mflop} / \mathrm{s}$ and a 32 Mbyte main memory, which is also organized as cache memory. The cells are connected by a unidirectional slotted ring network with a bandwidth of 1 Gbyte/s. The machine has

* The compiler strategy has recently been successfully implemented in a new compiler [2].

+ Upgraded to a 64-cell system after this experiment was performed. 


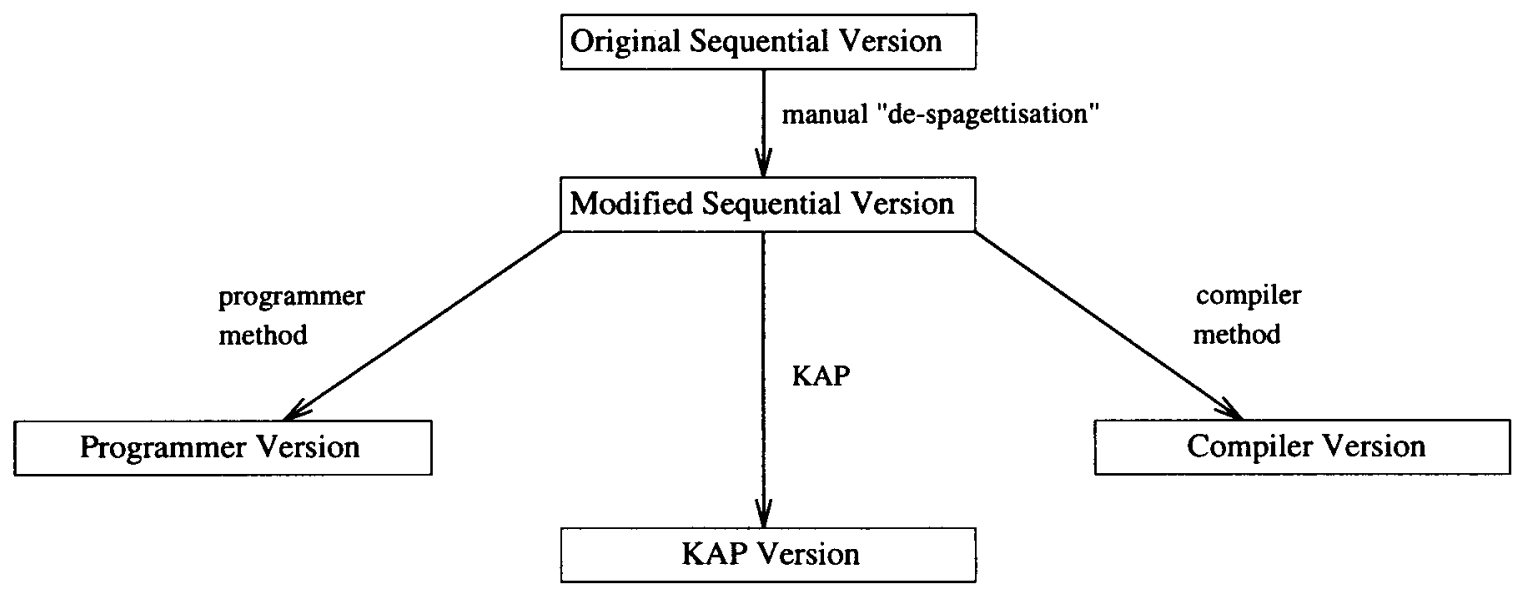

FIGURE 1 Relationship between versions of TRED2.

a Unix-compatible multiuser distributed operating system.

The memory system, called ALLCACHE, is a directory-based system which supports fully cache coherent shared virtual memory (SVM). Data movement is request driven; a memory read operation which cannot be satisfied by a processor's own memory generates a request which traverses the ring and returns a copy of the data item to the requesting processor; a memory write request which cannot be satisfied by a processor's own memory results in that processor obtaining exclusive ownership of the data item, and a message traverses the network invalidating all other copies of the item. The unit of data transfer in the system is a subpage which consists of 128 bytes (16 eight-byte words). The operating system manages page migration and fault handling in units of 16 Kbyte.

The KSR-1 processor has a level 1 cache. known as the subcache. The subcache is 0.5 Mbyte in size, split equally between instructions and data. The data subcache is two-way set associative with a random replacement policy. The cache line of the data subcache is 64 bytes (half a subpage).

There is a 2-cycle pipeline from the subcache to registers. A request satisfied within the main cache of a cell results in the transfer of half a subpage to the subcache with a latency of 18 cycles $(0.9 \mu \mathrm{s})$. A request satisfied remotely from the main cache of another cell results in the transfer of a whole subpage with a latency of around 150 clock cycles $(7.5 \mu \mathrm{s})$. A request for data not currently cached in any cell's memory results in a traditional, high latency, page fault to disk.
In order for a thread to access data on a subpage, a copy of the page in which the subpage resides must be present in the cache of the processor on which the thread executes. If the page is not present, a page miss occurs and the operating system and ALLCACHE system combine to make a copy of the page present. If a new page causes an old page in the cache to be displaced, the old page is moved to the cache of another cell if possible. If no room can be found for the page in any cache, the page is displaced to disk. Moving a page to the cache of another cell is much cheaper than paging to disk.

\subsection{Parallel Programming in Fortran}

Parallel execution of programs is achieved by allowing a number of threads to participate in the execution. Each thread is a flow of control within a process. By default, the threads are scheduled by the operating system, and may time-share on a cell with other threads, or be rescheduled from one cell to another during program execution. However the allocate_cells command can be used to reserve a number of cells for the execution of a program. Provided the number of threads required does not exceed the number of cells allocated, no time-sharing or movement of threads will occur.

Threads are managed in a Fortran program via a set of extensions to Fortran 77 consisting of compiler directives and library calls. For full details see $[10]$. The two most important directives are parallel region and tile. The parallel region directive encloses a section of code thus: 
c*ksr* parallel region ([options])

[section of code]

$\mathrm{c} * \mathrm{ksr} *$ end parallel region

The enclosed code is executed by a number of threads, which is specified as one of the options to the directive. In addition it is possible to declare scalar variables as private variables: each thread will then have its own copy of these variables. All other variables are shared between threads. In order for threads to identify themselves, the integer function $i p r_{-}$mid ( ) is provided, which returns a thread ID in the range $0, . . . p-1$ when there are $p$ threads executing the parallel region.

Synchronization, by semaphores, between threads can be achieved at the most basic level by calls to gspwt (get subpage wait) and rsp (release subpage) which, respectively, attain and release atomic ownership status on a specified subpage. If a subpage is in atomic state, a thread will block on a gspwt call until atomic status is released by another thread using rsp. Library routines are available which use these constructs to implement mutual exclusion (mutex) locks. condition variables, and barrier synchronisation. Barrier synchronization also occurs at the beginning and end of a parallel region. This mechanism is used by the compiler method to achieve parallel execution.

The parallel region, together with the synchronization mechanisms described above, is a very powerful parallel construct, but it requires careful management by the programmer and may necessitate significant code changes from programs requiring complicated scheduling of parallel work. Since loop-based parallelism is very common, a separate directive (tile) is supplied which applies only to perfect rectangular loop nests, including, or course, single loops. This reduces programmer effort, and the code changes required, for a class of common parallel constructs. The tile directive takes the following form:

c*ksr* tile (index list, [options])

[loop nest]

c*ksr* end tile
This divides the iteration space of the loop nest into a number of rectangular pieces (tiles). These tiles are then scheduled to be executed in parallel. The index list allows the programmer to specify which iterators are to be tiled. The options allow specification of the number of threads to be used and a choice of scheduling strategies. There are two strategies which are of interest in this experiment: slice and mod. The slice strategy divides the iteration space into $p$ roughly equally sized tiles. The mod strategy divides the iteration space into more than $p$ tiles (where possible) and schedules them on $p$ threads in a modulo fashion. For either strategy the size of the tiles can be fixed by the programmer or determined at run-time. In the latter case the tile size will normally be chosen as a multiple of 16 to help avoid false sharing of subpages. False sharing is said to occur when two or more threads write to different words on the same subpage. causing unnecessary data movement. The options also allow scalar variables to be declared as private variables, or as reduction variables. In the latter case results are accumulated in local copies of the variable, and code is generated which reduces these to a single variable at the end of the tiled loop.

A further mechanism for avoiding false sharing is the subpage directive, which forces a scalar variable, or an element of an array, to be aligned on a subpage boundary. In order to minimize data movement, it is sometimes advantageous to force several different tiled loop nests which have common iterators to be tiled with the same strategy, so that any value of the iterator is always assigned to the same thread. This facility is provided by the affinity region directive.

\section{APPROACH}

\subsection{Compiler}

The strategy employed by the compilation approach is intended to be generic for all distributed memory architectures. In particular, the strategy was not determined by experiment on the KSR-1, but by considering common overheads such as synchronization and communication. This will be in marked contrast to the method employed by a programmer.

A linear algebraic representation of the program is used which allows the easy formulation of transformations to optimize program behavior. The compilation strategy can be broken into two parts: (i) transforming the program to expose 
maximum parallelism and (ii) mapping this parallel work so as to utilize machine parallelism and minimize overheads. To expose parallelism, it is assumed that a parallelization tool such as Parafrase, Parascope, or tiny will be used. For the purpose of this experiment a method based upon accurate data dependence analysis, loop distribution, and scalar expansion was employed. The method is used in automatic vectorization as described in $[16$.

Once parallelism is exposed, the next stage is to map it to the parallel machine. This can be done in a computation or data-oriented manner. i.e., it is possible to partition parallel loops or partition arrays, scheduling them across the processors. The approach taken here is data oriented: data layout is determined in an attempt to be globally optimum. The layout determines the work to be performed on each processor and the amount of communication.

The compiler strategy is based upon a method described in [14] and was applied manually to the program. The cost model consists of three cost factors: load imbalance, interprocessor communication, and synchronization. The compiler optimization problem is to exploit sufficient parallelism while trying to minimize these costs. The most important feature is that there is a separation of concerns. The sequence of transformations the compiler undertakes reflects the perceived relative importance of hardware costs. Optimizing for one cost followed by another may miss an optimum that would be found considering them both jointly, but it makes compiler decisions tractable and avoids backtracking. This compiler strategy will be modified in the light of this experiment. It is hoped that an important product of this experiment will be to show to what extent generic compilation techniques are useful and what modifications are needed to successively utilize SVM in general and the KSR-1 in particular.

\section{Model of Computation}

The model of computation used is single process multiple data (SPMD) where each thread executes the same program, but operates on different sections of the distributed array. One thread is scheduled to each processor. A local-write rule is used where only data that are local to a processor/ thread are written to. This is verv similar to the owner-compute rule but is slightly more relaxed in that it allows exploitation of reduction parallelism.

To illustrate this point, consider the loop in Figure 2. If an owner-compute rule were used on this

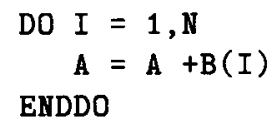

FIGURE 2 Simple reduction example.

program then the processor owning element $A$ would access all of $B$ and perform the calculation. With the local-write rule, each processor may calculate a partial sum which is then read by the processor owning B. Only local writes have taken place, but the parallelism associated with reduction has been utilized.

An essential characteristic of this approach is data affinity. Knowing where data reside allows the scheduling of work so as to minimize communication. The local-write rule is an example of this-work that writes to a particular piece of data is scheduled to the corresponding processor. Knowledge of the layout of data combined with an affinity approach can also help reduce interprocessor communication and the number of synchronization points.

Finally a compiler strategy must be scalable if it is to be generally applicable. Any compiler-introduced temporaries must fit in the appropriate part of the memory hierarchy for all data and problem sizes. Clearly, having a copy of all array data in every processor is not a scalable approach. The general strategy is as follows:

Preprocess. A program may contain GOTOs and induction variables which make analysis difficult. At this stage control flow normalization and induction variable recognition are performed so as to aid the parallelization and subsequent stages. If an array access is a simple (linear) function of a DO loop then there exists a large body of techniques to analyze and transform the enclosing loop. Sometimes, an array access is a linear function of a loop but it is hidden by an aliasing variable. Recognizing such aliasing of iterators is called induction variable recognition and, in itself, does not improve program performance, but does enable subsequent transformations.

Parallelize. Only loop parallelism is investigated. Transformations for parallelism are based upon accurate dependence information and loop distribution. An enormous amount of research has been undertaken in this area $[9,12,17,18,19]$, and there still remains much to do. No original methodology is claimed, the approach taken for the purposes of the experiment was based on tra- 
ditional vectorizing compiler techniques. After the program has been "cleaned up" by removing $\mathrm{GO}$ TOs, loop distribution was aggressively used to expose parallelism. Distributing a loop containing two statements results in two new separate loops each with one statement. Loosely speaking. reducing the number of statements in a loop increases the probability that it may be executed in parallel. The main restriction on parallelization is data dependence. While flow (or true) dependence cannot be removed, scalar expansion and statement reordering can be used to remove some output and anti-dependencies which are due to storage reuse rather than any flow of information. At this stage only uncovering of parallelism is addressed; there is no regard to how much overhead this may lead to, as this is the concern of later stages.

Align. In the compilation model used, data are eventually allocated to a processor. Alignment is concerned with the relative orientation of arrays with respect to a common index space and with maximizing the number of references that are local. If two arrays are said to be aligned on an index, then no matter what the form of the subsequent data partition, corresponding elements of each array will be mapped to the same processor. For example, consider the arrays $\mathrm{A}(10,10)$. $B(10,10)$. If they are aligned, then elements $A(1,1)$ and $B(1,1)$ will map to the same processor as will elements $A(X, Y), B(X, Y)$ for any values of $X$ and $Y$ in the range 1 to 10 . If a statement of the form $A(I, J)=B(I, J)+$. . exists in the program then this alignment will imply that no nonlocal communication of $B$ will take place. The processor that is writing to $A(I, J)$ will have $A(I, J)$ local to it due to the local-write implementation, and as $B(I, J)$ will be mapped to the same processor as $A(I, J)$, it too will be local. Therefore, at compile-time, we know there will be no communication necessary. If however. the statement were of the form $A(I, J)=B(J, I)+$ . then communication would take place given the above alignment. If, however, B were stored transposed relative to $A$ then again no communication would be needed. Determining the best relative storage to minimize communication throughout the program is the concern of this stage.

Partition. To determine the overall data partition, parallelism and overheads are examined, each of which will suggest a particular data parti- tion for a program section. In [14] four techniques are described which suggest a particular data partitioning to minimize a particular cost. The relative significance of each cost will determine a particular partitioning. In general there is a trade-off-each cost will suggest a conflicting data layout. The availability of certain post partition transformations may also have an effect on partitioning and must be considered where appropriate. The basic approach is based on concentrating any cost analysis on the "most significant" parts of the program and determining when a cost or transformation will have any significant impact. Given all this local information for "significant region" a global decision is made.

Synchronization. Synchronization can be a significant overhead in parallel programs. It should be proportional to the amount of communication. but naive analysis can lead to the insertion of excess synchronization points. This is often the case where barrier synchronization is used to ensure that data dependence is honored. It is possible to exploit the SPMD model of computation used in this strategy to reduce the amount of synchronization. By detecting those dependencies that are entirely local, synchronization points can be removed.

Mapping. After the data layout has been determined, it is necessary to generate the parallel program based on this decision. As this is an SPMD implementation, there is only one fork which occurs at the beginning of the parallel region. To ensure a local-write rule, only one thread ever writes to any particular array element. Essentially. the range of the iterators in a thread is limited by the bounds of the array elements written to. If there is any sequential work to perform, one thread will execute, while the others wait at a later synchronization point. This approach prevents the high cost of forks and data movement. but is less dynamic. Whilst data cannot be statically allocated to processors on the KSR-1, threads can be. If only one thread writes to a piece of data that data will be allocated at the site of that thread and will remain there as no other thread will ever wish to have ownership of it. Hence it is possible to exploit data locality based on alignment and layout on a COMA architecture.

Nonlocal Data Reuse. Once a nonlocal array element has been referenced it is desirable to store it locally if it is to be referenced again. Such a 
scheme exploits temporal locality by detecting data reuse in the program. Much research in compiler restructuring and architecture design has been directed at providing such a facility. Essentially by examining array references, it is possible to determine if data access is invariant of an iterator or combination of iterators. For a fuller description of this method see [15]. In this article, the data reuse method is extended by considering only those array accesses that are known to be nonlocal. Perfectly aligned data will be local and need not be considered.

Each of the above stages is applied in sequence to give a parallel FORTRAN program, as shown in Figure 3.

\subsection{Programmer}

The objective in this experiment, in common with much scientific parallel programming, is to minimize the parallel execution time for an existing piece of sequential code. This minimization exercise is, in general, constrained by the amount of programmer effort available. In this study, an attempt is made to produce an efficient parallel implementation while keeping code changes, and therefore programmer effort, to a minimum. The methodology described here has been developed during approximately 18 months of programming experience on the KSR-1. The results of applying

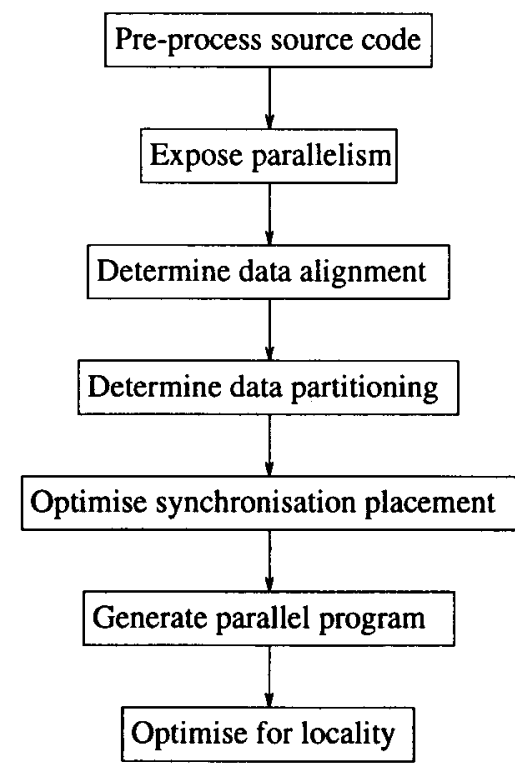

FIGURE 3 Flow diagram for compiler approach. this methodology to large-scale applications are reported in [6] and [7]. Although in some respects, notably in certain cures for some performance bugs, the approach is machine specific, much of it will be applicable to other SVM architectures. It is, however, notable different from the approach that is required on traditional, messagepassing, distributed memory architectures.

The approach taken here is one of incremental parallelization, and relies heavily on the use of profiling and program instrumentation. The programming model adopted is essentially a shared memory model, where by default all data are globally accessible. All code is executed by a single thread (the master thread), unless the code is enclosed in a parallel construct, such as a paralle1 region or tile directive (see Section 2.2). The master thread controls the synchronization necessary at the beginning and end of each parallel construct.

The approach can be thought of as a progressive reduction in parallel overheads. For a program running on $p$ processors, the parallel overhead $O_{p}$ is defined as the difference between the actual execution time and that predicted by a naive Amdahl's Law model (where all code is assumed to be parallel), i.e.,

$$
O_{p}=T_{p}-\frac{T_{s}}{p},
$$

where $T_{s}$ is the execution time for the sequential version and $T_{p}$ is the execution time for the parallel version on $p$ processors. It is helpful to classify the overheads into a number of classes according to their source. There seems to be no general consensus as to how best to define this classification: see [5] and [4] for two examples of classification schemes. The classes used here are intended to be as program oriented as possible, and therefore largely hardware independent, because their purpose is to help identify the program constructs which are causing poor performance, rather than hardware bottlenecks. These classes are

1. Lnparallelized code.

2. Load imbalance in parallelized sections.

3. Memory access costs. These can be subdivided into remote memory accesses (movement of data between cells and local memory accesses (movement of data between levels of memory hierarchy within a cell). Note that memory access costs can either increase or decrease when the code is paral- 
lelized, so it is possible for negative overheads to occur.

4. Synchronization. This is the cost of barriers and locks, and of any idle time caused by contention for locks.

5. Scheduling. This is the cost of any computation necessary to determine at run-time which tasks are to be scheduled on which processor.

At any stage of the parallelization process, the strategy is to measure the contribution of the overhead in each of the above classes for a given problem size, and to identify the sections of source code responsible for these contributions. The programmer than attempts to reduce the most significant overheads by changing the parallel code. This process is then repeated until the programmer runs out of time or new ideas. Sometimes a point may be reached where no further progress seems possible. The programmer may then decide to backtrack to a certain point in the process, and try again in a different direction.

Naturally the programmer must take care that any transformation of the program preserves the program semantics. This is normally achieved by checking the results generated by a sample input data set. This is not entirely foolproof, however, as although incorrect results indicate an incorrect transformation, the converse is not true. A much more difficult issue is whether a code transformation can affect the numerical stability of an algorithm.

Initially, of course, all the overhead will be due to unparallelized code. Thus the first few steps of the parallelization process will consist of exposing and exploiting parallelism in appropriate sections of the sequential code. To aid this process, the programmer must first obtain an easily readable form of the code. The most important factor here is a simple control flow structure, with as many GOTO statements replaced by conditionals as possible. Nonstandard language features such as the DO WHILE statement are helpful here. Clear visual layout (indentation of loops and conditionals) and removal of induction variables are less important, but may be of assistance. Much of this work (except the removal of induction variables) can be automated using commercially available tools. Next the programmer must identify the important parts of the code; i.e., where most of the execution time is spent. In simple cases this can be done by inspection, but, in general, it is necessary to obtain a profile by code instrumentation.
The instrumentation can be in the form of a profiling tool, such as the standard Unix utility GPROF. or by hand-inserted calls to a timer routine. Usually the latter is more satisfactory, if more laborious. With profiling information in hand, the programmer sets out to expose parallelism in the most expensive sections of the code. In cases where the code consists mainly of loops, an automatic parallelization tool may be of some assistance. Current commercially available tools, however, can only apply a limited amount of restructuring, and for more complex loops, or where no loops are explicitly present, this process must be done by hand. Fortunately, programmers often have a very powerful tool at their disposal-they can reason about the problem at a higher level of abstruction in terms of the algorithm which the code implements. Parallelism may be obvious at this level even if it is deeply obscured at the code level, because at the algorithm level there is a more abstract, higher-level, description of tasks and data objects. It is often easier 10 identify independent tasks at the algorithm level, rather than sets of independent arithmetic operations at the code level. If the algorithmic structure is not available to the programmer, thanks to inadequate or unavailable documentation, then provided something is known about the abstractions employed at the algorithm level, reverse engineering of the algorithm may be possible.

Having exposed parallelism in a particular section of code, the programmer may be presented with a number of choices as to how to exploit it. Some of these options may be immediately rejected if it is obvious that they will incur unacceptably high overheads. Having done this, the choice will normally be determined by the amount of effort involved in terms of changes to the code. Further analysis of overheads will decide on whether more effort on this section of the program might be beneficial.

Once unparallelized code is no longer the dominant source of overhead, programmers can turn their attention to reducing overheads in the parallel sections of code. The part of the parallelization strategy which is causing the most overhead is targeted for alteration. The programmer now has 10 find an alternative strategy that will reduce the total overheads. This is largely a matter of reasoning about the program (possibly at the algorithm level) using a rough cost model for overheads, combined with a certain amount of inspiration. While it is impossible to give a general "recipe" for parallel programming, there are certain tech- 
niques, some generally applicable, others architecture specific, which often prove useful in reducing the various sources of overhead.

\section{Load Imbalance}

Load imbalance may be either due to processors having different amounts of arithmetic operations to do, or to processors spending different amounts of time in memory access operations. In the former case, block interleaving of loop iterators is a common solution, provided that the number of computations is a suitably smooth function of the iterator. If it is not smooth, then it may be necessary to devise an explicit scheduling strategy. Most run-time self-scheduling policies, the simplest of which is first-come-first-served, are rarely a success in scientific applications, since they tend to destroy data locality.

There may be a number of causes for imbalance in memory accesses. If the pattern of imbalance changes from one instance of the loop to the next then it is often a symptom of false sharing; see later for possible cures. If the pattern is repeated from one instance of the loop to the next then it may be a genuine requirement of the algorithm, in which case the techniques used to balance computations may be applied, though it should be noted that there will be a tradeoff between remote access and load imbalance, and the optimum solution may not be load balanced.

Another possibility is that the imbalance is a result of cache interference misses being much more severe for some values of the iterator than for others. It may be possible to reorganize data structures to avoid this.

\section{Remote Memory Accesses}

If significant numbers of remote memory accesses are occurring then it will be necessary to determine whether they are genuinely required by the parallel algorithm, or whether they are a result of false sharing. In the KSR-1 memory sustem, false sharing can occur at both the subpage level and the page level. False sharing of subpages can be overcome by padding and aligning of arrays (or scalars) to ensure that no two threads access different words on the same subpage. It may also help to avoid tiling over the first index of arrays, if possible, or to use tile sizes which are a multiple of the subpage size. In order for a thread to access a subpage, the page on which the subpage lies must be resident on the requesting cell. False sharing at the page level can result if many threads access subpages that reside on the same $16 \mathrm{~K}$ page. Since all the threads accessing a page must have space allocated for that page. capacity misses may cause pages to be displaced to other cell memories, with a resulting cost when they are next required. The solution to this problem is to reorganize the data structures or the parallelization strategy such that. as far as possible, each thread accesses data which occupy a contiguous region of the virtual address space.

If genuine communication is a significant source of overhead, then it will be necessary to reorder computations to improve locality. For example, if the parallization strategy consists of a spatial decomposition of a regular grid. with neighbor-wise communication. then choosing subdomains with a minimum boundary length mav reduce the number of remote data accesses. A frequent source of communication on SVM systems is sequential sections of code which may account for a small proportion of the run-time in the sequential version, but cause a significant number of remote accesses in the parallel version because data are gathered by the master thread and subsequently scattered again.

\section{Local Memory Accesses}

Local memory access overheads are normally a result of the parallelization strategy causing a loss of locality in the subcache. The solution is to reorder the computations so as to maximize the reuse of data items that are loaded into the subcache. In scientific codes with large arrays, ensuring strideone memory accesses through the arrays is frequently of significant benefit.

\section{Synchronization}

If synchronization costs dominate, then the programmer should check whether all synchronization points are indeed necessary to ensure that the program generates correct results. The KSR Fortran ti le directive implies a barrier synchronization at the beginning and end of the tiled loop nest. These barriers may not always be necessary, particularly if the tiled loop is contained in an outer loop. Loop fusion can be used to reduce the number of synchronization points, as can the parallel region directive combined with calls to barrier routines.

If locks are used, then overheads can result both from the actual calls to the lock routines, and from any contention for the locks. If contention is significant, then it pays to reduce the length of any 
critical sections as much as possible. If on the other hand the overhead arises largely from the calls themselves, it may be beneficial to reduce the number of locks, even if this means increasing the length of the critical section. Note that on the KSR locks are implemented via atomic status bits on subpages. Accessing these directly is significantly cheaper than using the mutex lock routines.

\section{Scheduling}

Although run-time scheduling is convenient, as it allows the number of threads to be varied without recompiling, it can be costly. On the KSR-1, scheduling can contribute at least as much to the cost of the tile directive as does barrier synchronization. This can be minimized by supplying as much information as possible to the run-time system, or even by doing the scheduling in user code, using the parallel region directive.

Tradeoff situations are very common-reducing one source of overhead frequently results in the increase of another. Furthermore, the sources of overhead may not be localized in one section of the code-changes to one section may affect the overheads in another. In such situations it will be necessary to run experiments to determine which parallel strategy is the best. The run-analyzemodify process can be iterated, including possible backtracking, until either performance is satisfactory or other constraints force it to come to an end, as illustrated in Figure 4.

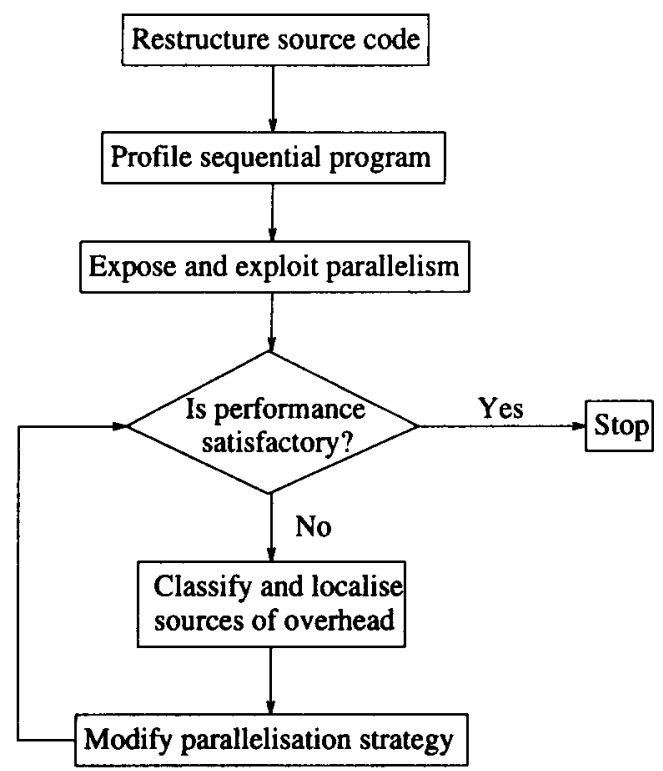

FIGURE 4 Flow diagram for programmer approach.

\section{COMPILER METHOD}

\subsection{Preprocessing and Parallelization}

A considerable amount of preprocessing must take place before TRED2 can be sensibly parallelized. Firstly, control-flow normalization is attempted where the GOTOs are replaced by conditionals, leaving the structure of the code as intact as possible. Secondly, induction variable substitution takes place, which is then followed by forward substitution of scalar temporaries which hold array values. For example, consider the program in Figure 5a, where $I$ and $L$ are induction variables. All occurrences of $\mathrm{I}$ are replaced by $\mathrm{N}+2-\mathrm{I}$ I and all occurrences of $\mathrm{L}$ are replaced by $\mathrm{N}+1$ - I I to give Figure 5b. A full listing of the code after this process has been applied is given in the Appendix.

Extracting parallelism by loop distribution is by no means trivial; while it is relatively easy to see, in Figures 5a and 5b, that the $\mathrm{K}$ loop will provide some parallelism and its two statements do not interfere, the II and J loops look sequential. This is, in fact, the conclusion of Gupta [8] when using the Parafrase environment. However, if certain transformations are applied, the program in Figure $5 \mathrm{c}$ can be obtained where the distributed $\mathbf{J}$ loop is also parallel.

To get to this form requires careful analysis of the order of access to each of the arrays. In Figure 5 a statements 2 and 3 , within the $\mathrm{K}$ loop, do not refer to each other and can thus be easily loop distributed to give Figure 5b. Each of the separate references to $\mathrm{Z}$ in the code fragment of Figure $5 \mathrm{~b}$ refers to a different region of the array. $Z(J, I)$ in the first line accesses the strictly upper triangular region of the $Z$ array. On the next line the read of $\mathrm{Z}(\mathbf{J}, \mathbf{J})$ is simply the diagonal, while the remaining read of $\mathrm{Z}(\mathrm{K}, \mathrm{J})$ is to the strictly lower triangular region of the $Z$ array. None of these accesses refer to the same region of the $\mathrm{Z}$ array and hence there is no order placed on the evaluation of the statements due to the data dependencies arising from the access to $\mathrm{Z}$.

There is no cross-iteration data dependence with respect to $G$, but there is a flow dependence from statement 1 to 2,2 to 3 , and 3 to 5 , therefore the lexicographic order $1<2<3<5$ must be maintained. G must be scalar expanded before loop distribution can take place to allow each iteration of the distributed $J$ loops to refer to the correct value of $\mathrm{G}$.

The only remaining variable preventing distri- 


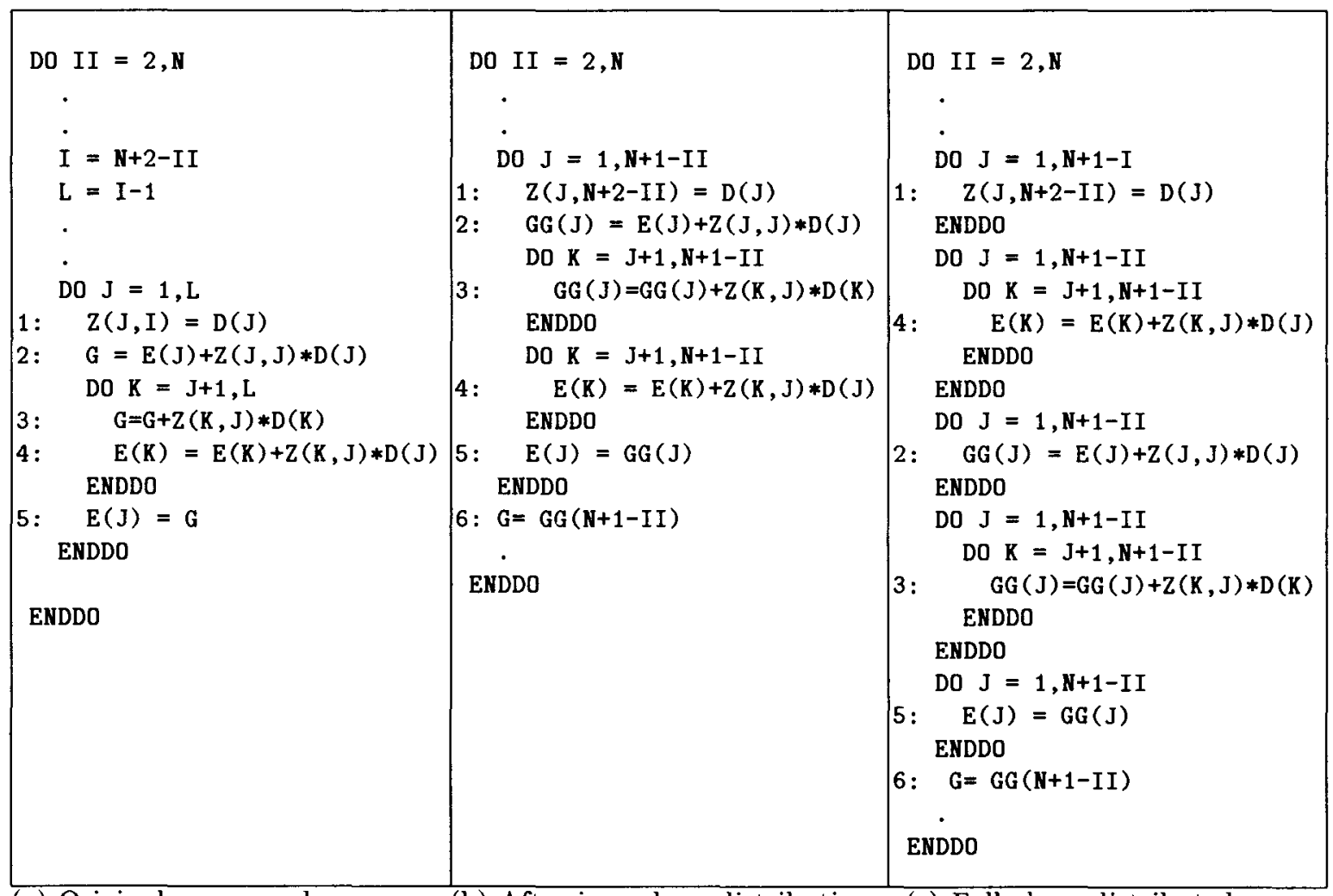

(a) Original source code.

(b) After inner loop distribution.

(c) Fully loop distributed.

FIGLRE 5 Parallelization.

bution of the $\mathrm{J}$ loop is the variable $\mathrm{E}$. There is cross-iteration antidependence from statement 2 to 4 . The values written in statement 4 are read by statement 2 on the next iteration of the $\mathrm{J}$ loop. If loop distribution were performed then statement 4 would have to precede statement 2 (see $[14]$ ). The output dependence from 4 to 5 implies that statement 4 must precede $\overline{5}$ if $J$ were distributed. This gives two partial orders on the statements. $\mathrm{G}$ requires $1<2<3<5$ and $E$ requires $4<2<5$. Fortunately these orders are not conflicting giving a legal order (amongst others) of 1, 4, 2, 3. 5. The final loop distributed version is shown in Figure $5 \mathrm{c}$ where it is easy to determine that each of the loops is parallel. Any one of these stages in itself is not very complex. However, the correct ordering of analysis and transformation is difficult and often defeats parallelizing compilers.

\subsection{Alignment}

Alignment has direct global impact: the location of a value must be consistent throughout its potential multiple usages in a program. In this experiment, a restricted method is used whereby only index reordering is considered. Alignment is NPcomplete [11], so to reduce the complexity of deciding the overall alignment, only the most significant regions of the program are examined. These are the regions where the most communication and computation are expected and correspond to the deepest loop nests and largest arrays. In the presence of conditionals static estimates based on control-flow information are used to determine the significant regions. A global alignment graph is created where an are between two indices indicates that it is desirable that the indices be aligned. After applying a variant of the index domain alignment algorithm [11], the following global alignment, using a linear algebraic representation. was determined for the referenced arrays $E, Z, D$, and $G G$.

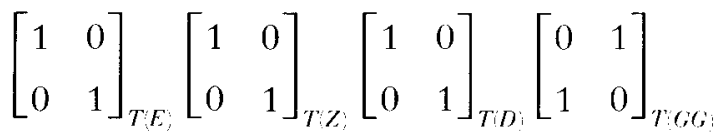

This indicates that the one-dimensional arrays $\mathrm{E}$ and $D$ should both be aligned with the first index of $\mathrm{Z}$ while GG should be aligned with the second. 


\subsection{Global Data Partitioning}

Partitioning analysis is applied to all the significant statements, where each statement will suggest a particular data partition. The most popular partition(s) are stored in a set. Para is the set of the most popular partitions if the parallelism analysis is applied, Align is the corresponding set based on alignment, Load that on load balance, and Region that on the region of access. The region of access analysis tries to determine the best data partition based on the assumption that data reuse transformations will take place after partitioning.

Table 1 shows the results of applying each form of analysis for the TRED2 code based on the significant regions a,b,c,d,e (see code in Appendix). Here 1 denotes partitioning by the first index of $z$, 2 by the second index, $1 \wedge 2$ by both indices, and $\emptyset$ by neither. As far as parallelism is concerned. all partitions were equally acceptable. The load balance analysis found no suitable iterators, while alignment preferred either the first index or the second index but not both. Finally the region of access preferred index 2 or indices 1 and 2. Applying the trade-off algorithm [14] to the TRED2 example gives the result that data should be partitioned along the second index. None of the analyses gave a unique choice, implying the certain sections have conflicting requirements. Other programs will have different behavior. Because no one data partition is clearly superior throughout the whole program, it seems likely that the partitioning decision in this program is not crucial, and other factors such as later optimizations may dominate. Once the global partition has been decided it is necessary to generate the code that each thread will execute. The remainder of this section describes the code generation and subsequent postpartitioning transformations.

Table 1. Partition Decisions for Each Analysis

\begin{tabular}{|c|c|c|c|c|}
\hline Stat. & Para & Load & Align & Region \\
\hline $\mathrm{a}$ & $1,2,1 \wedge 2$ & $\varnothing$ & 1 & 1 \\
\hline b & $1,2,1 \wedge 2$ & $\varnothing$ & 2 & 2 \\
\hline $\mathrm{c}$ & $1,2,1 \wedge 2$ & $\varnothing$ & 1 & $1 \wedge 2$ \\
\hline d & $1,2,1 \wedge 2$ & $\varnothing$ & 2 & 2 \\
\hline e & $1,2,1 \wedge 2$ & $\varnothing$ & $1,2,1 \wedge 2$ & $1 \wedge 2$ \\
\hline \multicolumn{5}{|c|}{ Indices } \\
\hline 1 & 5 & 0 & 3 & 1 \\
\hline 2 & 5 & 0 & 3 & 2 \\
\hline $1 \wedge 2$ & 5 & 0 & 1 & 2 \\
\hline
\end{tabular}

\subsection{Synchronization}

Synchronization between threads was achieved using a barrier synchronization method. A call to a subroutine Lock is inserted at each synchronization point, in which each thread atomically increments a shared counter. After this "checkingin," each thread calls a subroutine Spin. where it spin-waits until a synchronization variable is unset by the last thread exiting the subroutine Lock. At the time hand-coded routines were used but it seems that the system barrier routines mav actually be faster. If a naive sunchronization method were employed, whereby a barrier is placed at the end of every parallel loop nest, then the number of synchronization points for the TRED2 code would be 36 . By detecting those dependencies that are entirely local, synchronization points can be removed reducing the total to 14 .

To illustrate this point consider the fragment in Figure 6 . The only synchronization point required is following the final ENDDO due to a flow dependence on GG $(\mathrm{J})$ from statement 3 to statement 5 . The flow dependencies from statements 1 to 2,2 to 3 , and 3 to 4 can be removed as all writes are local, the reads are aligned, and are therefore also local. Output dependence from statement 1 to 4 and 2 to 3 can be removed due to the SPMD implementation and finally the antidependence from 2 to 4 can be removed as the writes and reads are totally local.

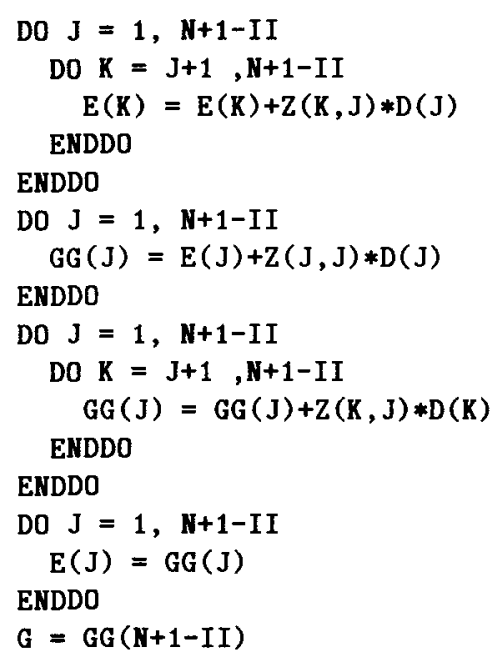

FIGURE 6 Synchronisation example. 


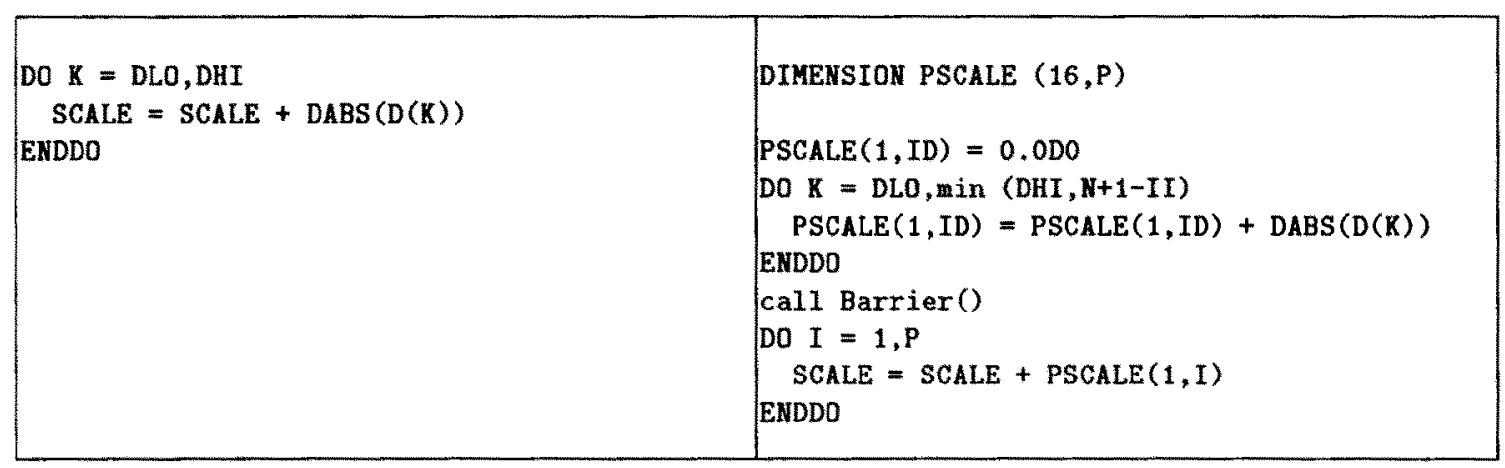

FIGLRE 9 Partitioned reduction.

boundary, and no two objects reside on the same subpage. This helps remove false sharing by ensuring that partitioning of data occurs along subpage boundaries. Reduction parallelism was exploited in a modified manner to that described in [13] so as to avoid false sharing. The source program shown in column 1 of Figure 9 is translated into the form in column 2.

Each thread writes the partial sum of the locally available elements of $D$ into its local element of the global PSCALE array. PSCALE is expanded to a $16 \times \mathrm{P}$ array to prevent false sharing. Sixteen elements reside on a subpage, and this expansion can be seen as padding the array such that each thread writes to a separate subpage. Without this expansion, up to 16 processors would be trying to gain write ownership of one subpage at any one time. Once each partial sum is calculated every processor accesses every other processor's partial sums and accumulates them into a local private copy of the scalar scale. Thus the reduction parallelism available has been exploited without incurring false sharing at the expense of one barrier.

\subsection{Data Reuse}

Once a nonlocal array element has been referenced, it is desirable to store it locally if it is to be referenced again. Such a scheme exploits temporal locality by detecting data reuse in the program. For a fuller description of this method see $[15$. In this article, the data reuse method is extended by considering only those array accesses that are known to be nonlocal. Perfectly aligned data will be local and need not be considered. For example, consider the program fragment shown in column 1 of Figure 10.

References $D(J)$ and $E(J)$ are local as they are aligned with the second index of $\mathrm{Z}$. The nonlocal references $D(K)$ and $E(K)$ are invariant of iterator $J$. On applying the data reuse transform, the program shown in column 2 is derived. This reduces the number of nonlocal array accesses by $2 \times$ $(Z H I-Z L O+1)$. The applicability of the region of access analysis depends on the ability to exploit data reuse. This postpartition transformation is therefore integral to the partitioning algorithm described earlier.

\section{Spatial Locality}

The main shortcoming of the compiler approach is due to inaccuracies in the assumed architecture model. Divergence has already taken place when considering false sharing in compiling for reduction parallelism. While the KSR-1 is a distributed memory machine with a single address space it

\begin{tabular}{|l|l|}
\hline DO $J=Z L O, Z H I$ & DO $K=Z L O, N+1-I I$ \\
DO $K=J, N+1-I I$ & $T D=D(K)$ \\
$Z(K, J)=Z(K, J)-D(J) * E(K)-E(J) * D(K)$ & $T E=E(K)$ \\
ENDDO & DO $J=Z L O, \min (Z H I, K)$ \\
ENDDO & $Z(K, J)=Z(K, J)-D(J) * T E-E(J) * T D$ \\
& ENDDO \\
\hline ENDDO
\end{tabular}

FIGURE 10 Data re-use transformation. 
also has the additional features of cache-only. SVM.

The compiler model assumes that the only communication overhead is between processors, and that it is a simple function of the amount of remote data accesses. Both these assumptions are not correct. The main impact on the compiler strategy is that striding through memory in the correct direction is important. In other words, spatial locality is significant, but is currently completely ignored in the data reuse section, where only temporal locality is considered. Furthermore, the main overhead is not due to increased interprocessor communication, but rather because of large intraprocessor communication. Not taking stride into account means that there will be a subcache miss when accessing each element of $\mathrm{Z}$ in four of the five significant regions. The main memory, or cache, is large enough so that, even when striding in the wrong direction, the cache lines (or subpages) will remain local until reused. However, this is not true of the subcache, which is much smaller.

Ideally interprocessor and intraprocessor communication should be optimized using a hierarchical memory model as described in [15]. However, since at present this often induces excessive loop overhead, a more simplistic method was used, where loops are reordered such that the innermost iterator strides through memory with a step of one. Loop interchange can be applied on all the statements with stride problems and is a postpartition transformation, i.e., it does not affect any of the previous sections. In particular it has no effect on data partitioning and mapping. This technique was used, with and without interleaving, to give two new programs. Overall the data sizes interleaving proved expensive. Interleaving should have improved performance, but it seems that the overhead due to the mod operator removed any benefit due to better load balancing. A more efficient implementation of interleaving may give better results, but due to time constraints, this was not further investigated in this article.

\section{PROGRAMMER METHOD}

Starting Point: Control flow normalized code with variable substitution, produced as described in Section 4.1. Working from a control-flow normalized version is important as it is much easier to build a mental picture of the control structure of the code. The variable substitution is less important, but it allows an easier evaluation of memory access patterns.

Step 1: Identify the "important" sections of the code.

TRED2 has no subprograms, so this step reduces to identifying the loop nests which take the longest time to execute. This is achieved by adding timing routines round every loop in the code. There are two loops at the outermost level. Both contain significant amounts of work, but a cursory examination shows that data dependencies exist between iterations of these loops. Both are sufficiently complex that restructing the code to expose parallelism would be a formidable task. If this is indeed possible, it could only take place without unreasonable effort by restructuring at the algorithmic level, as the complexity of analysis and transformation required at the code level would defeat all but the most dedicated programmer. At the next level of loop nest, three loop nests account for over $99 \%$ of the execution time on a single processor for problem size $N=400$. These are shown in Figure 11.

The inner (K) loops in Fragments 2 and 3 (but not that in Fragment 1) could be parallelized as they stand. However, the timing results and a naive operation count suggest that unless $N$ is very large (many thousands), the granularity of these loops is too small for the KSR-1 to achieve any speedup, because a tile directive is implemented via barrier synchronizations at both the beginning and the end of the loop. (Here programmers are appealing to experience-they know that the overhead incurred in tiling a loop is $\sim 1 \mathrm{~ms}$. If a loop executes in less than $\sim 1 \mathrm{~ms}$ it is certainly not worth parallelizing.) In some circumstances the repeated synchronization is not necessary, and this is a drawback of the tile directive. In these cases, however, the synchronization would be necessary to ensure correct results. Thus, to achieve any parallel performance, the programmer must concentrate on parallelizing the $J$ loops.

Step 2: Try to expose parallelism with an automatic tool.

The automatic parallelization tool KAP is applied to the code and its treatment of each of the three fragments observed. KAP successfully parallelizes 
Fragment 1

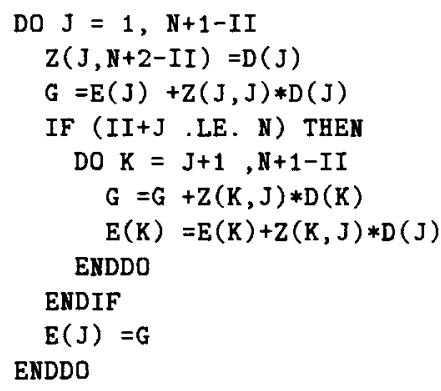

Fragment 2

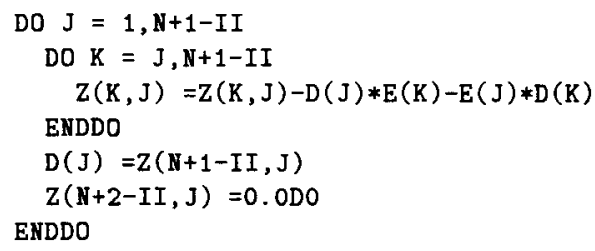

Fragment 3

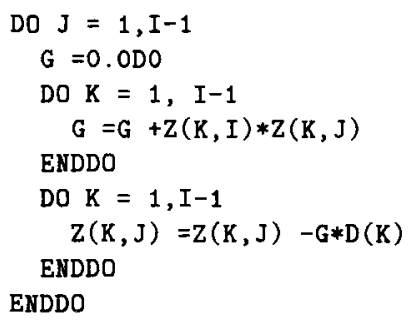

FIGURE 11 Important fragments in TRED2.

the $J$ loop of Fragment 3 and (after trying a variety of combinations of options) the $\mathrm{K}$ loop of Fragment 2, but nothing in Fragment 1.

Step 3: Exploit parallelism in Fragment 3.

Following KAP, a tile directive is added to the $\mathrm{J}$ loop of the third fragment as it stands, using the default strategy (slice). The scalars G and $\mathrm{K}$ are declared as private variables to ensure that each thread has its own copy of them. This reduces the execution time for this loop by a factor of 6.5 on eight cells for this loop $(N=400)$. The default choice of tile size, however, is always a multiple of 16 , and since the upper loop bound is increasing by one each time the loop is executed, this can result in some poor load balancing. Thus the tile size was set to be $\lceil i-1 / N\rceil$, giving a reduction in execution time of 7.7 times on eight cells for this loop.
Step 4: Expose parallelism in Fragment 2.

Attention is now concentrated on Fragment 2, as this appears easier to deal with than Fragment 1. The reason KAP fails to parallelize the $J$ loop is a data dependence on array $\mathrm{D}$. However, since each iteration of the $\mathrm{J}$ loop writes to $\mathrm{D}(\mathrm{J})$ but subsequent iterations only read $D(J+1) \ldots D(N-$ 1+II), the loop can be split as shown in Figure 12.

The double-nested loop, which contains most of the work in the fragment, is now clearly parallelizable. Based on the known cost of tiling a loop. the other loop contains too few arithmetic operations to be worth parallelizing, and so remains sequential.

Step 5: Exploit parallelism in Fragment 2.

The new double loop has a triangular iteration space, and therefore slice strategy would result in load imbalance. Mod strategy (which performs a block interleaved partitioning of the loop) is used to improve the load balance. The tile size is chosen by experiment: although a tile size of 1 would give the best load balance, there is an overhead associated with tile generation which is proportional to the number of tiles. Thus the programmer searches for the value which minimizes the execution time for this loop. For $\mathrm{N}=400$, values in the range 1 to 20 are considered, as it is soon apparent that the optimal value lies between these two. Unfortunately there are now some nonlocal memory accesses to the array $\mathrm{Z}$, because the partitioning strategies for Fragment $2(\bmod )$ and Fragment 3 (slice) conflict. Since the dominant overhead is now the unparallelized code of Fragment 1 , the programmer refrains from any further optimization of Fragment 2 at this stage. All three

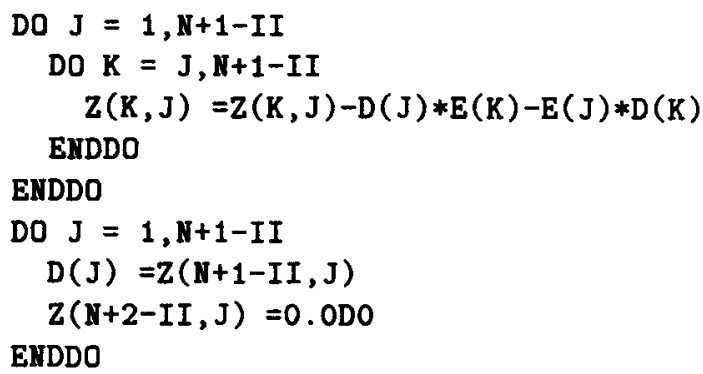

FIGURE 12 Fragment 2: parallel version. 
fragments reference the two-dimensional array $\mathrm{Z}$, which is by far the largest data object in the program, so the programmer will want to choose tiling strategies for all three fragments which give sensible data access patterns for $\mathrm{Z}$.

Step 6: Expose parallelism in Fragment 1.

Fragment 1 is the hardest of the three to parallelize; it is not at all easy by inspection to spot a transformation which exposes parallelism. However the problem can be simplified with the following observations:

The statement

$\mathrm{Z}(\mathrm{J}, \mathrm{N}+2-\mathrm{II})=\mathrm{D}(\mathrm{J})$

writes to column $\mathrm{N}+2-\mathrm{II}$ of $\mathrm{Z}$, but the rest of the loop only writes to columns 1 to $N+1-I I$. Also the array $D$ is not written to in this fragment. Thus this statement can be distributed into a separate loop.

The conditional

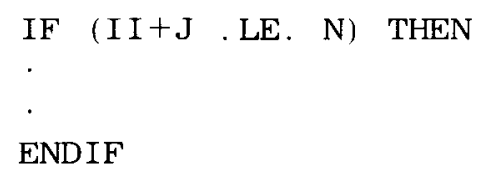

is superfluous, because it is implied by the bounds of the loop it contains.

Thus the fragment can be rewritten as in Figure 13 and the programmer can concentrate on the second $J$ loop. It is still not clear how to proceed from here, so a decision is made to view the problem at the algorithm level rather than at the code

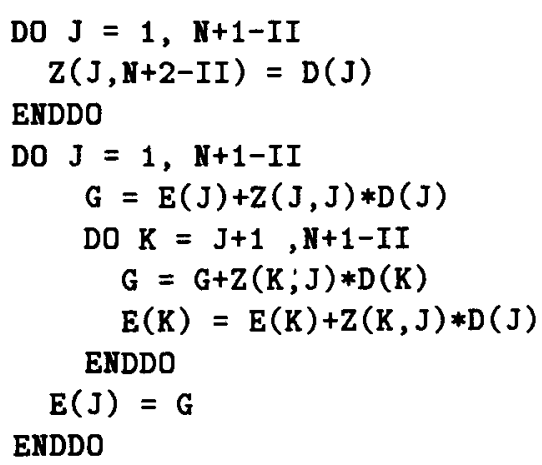

FIGURE 13 Simplified Fragment 1.

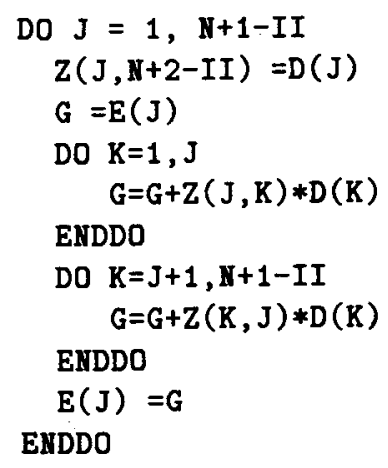

FIGURE 14 Fragment 1: parallel version.

level. This is a linear algebra kernel code, so it may be possible for the programmer with sufficient experience in this area to work out what this loop is doing in terms of matrix/vector operations. A "paper run" of the fragment with $\mathrm{N}=4$ is carried out, and the pattern of accesses to E, D, and $\mathrm{Z}$ leads the programmer to recognize that this loop has the form of adding a matrix-vector product to a vector. If the subarrays $D(1: N+1-$ II $)$ and $\mathrm{E}(1: \mathrm{N}+1-\mathrm{II})$ are thought of as vectors $d$ and $e$, then this loop has the form $e:=e+Y d$ for some $Y$. Further inspection shows that if the square subar$\operatorname{ray} Z(1: N+1-I I, 1: N+1-I I)$ is thought of as a matrix $Z$, and the strictly lower triangular part of $Z$ denoted by $L$ and the diagonal part by $D$, then $Y=L+D+L^{T}$. It is now evident that this loop is parallelizable, since the product of each row of the matrix $L+D+L^{T}$ with $d$ can be computed independently. With this in mind, the fragment is rewritten as in Figure 14, where the two $J$ loops have been fused together again. The $J$ loop can be parallelized. Note that use of $\mathrm{G}$ here as a temporary variable is not necessary to exploit parallelism. However, it prevents false sharing of subpages of the array E. Note that this is the only one of the three fragments where false sharing is a potential problem, since it is the only one where writes to a one-dimensional array occur. In the other two fragments all the writes in parallel loops are to $\mathrm{Z}$, and since it is the second index which is partitioned, there is no possibility of significant false sharing.

Step 7: Exploit parallelism in Fragment 1.

A tile directive is added to the $J$ loop. Although this loop is load balanced in terms of floating point operations, it is not balanced in terms of local/ 
nonlocal memory accesses, because the $J$ th iteration makes $\mathrm{J}$ accesses to a row of $\mathrm{Z}$, which are mostly nonlocal, and $\mathrm{N}+1-\mathrm{II}-\mathrm{J}$ accesses to a column of $Z$, which are all local. Thus mod strategy is used to achieve block interleaving.

\section{Step 8: Data access patterns}

Use of timers, hardware monitoring information, and an event logging tool (GIST) shows that by this stage the overheads are no longer dominated by unparallelized code, but by load imbalance and remote memory accesses. Both of these can be reduced by considering data access patterns. The array $\mathrm{Z}$ is the only two-dimensional array, so it is only necessary to consider accesses to $\mathrm{Z}$ when optimizing for data alignment. In Fragments 1 and 2 it is necessary to distribute $\mathrm{Z}$ by interleaved blocks of columns in order to achieve load balance. Thus the obvious choice is to use interleaved blocks of columns for all three parallelized loops. To ensure that the same columns of $\mathrm{Z}$ are always accessed by the same processor, an affini ty region directive is added to the code, which forces the mod strategy to be used on all tiled loops inside it. The programmer also needs to choose a sensible block size. Experiment shows that a block size of 4 is optimal for $N=400$. Very small block sizes ( 1 or 2 ) give the best load balance, but produce substantial overheads associated with tile generation. For larger $\mathrm{N}$ the block size should be scaled with $\mathbf{N}$ so that the number of blocks is constant with problem size. Thus a heuristic block size of $\max (4, \mathrm{~N} / 100)$ is chosen. This could possibly be refined, given additional programmer effort.

Step 9: Alternative parallelization of Fragment 1.

A large proportion of the remaining overheads are associated with Fragment 1. At least part of the reason for this is that, having exposed parallelism, the loop

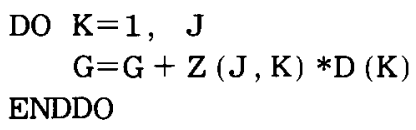

makes nonunit strides through array $\mathrm{Z}$, resulting in an increased subcache miss rate. In order to preserve unit stride through $\mathrm{Z}$, a complete rethink of the parallelization strategy for this fragment is undertaken, and an alternative strategy is chosen. It may be noted that this fragment computes addi- tive updates to the array E. It is therefore possible to accumulate the updates computed by each thread in a local copy of the array $\mathrm{E}$, and then perform a reduction operation to add in all the updates to $\mathrm{E}$ itself. In other words, the original $\mathrm{J}$ loop can be made parallel by making private copies of $\mathrm{E}$. This technique is frequently used in $\mathrm{N}$ body problems for computing the sum of forces on particles due to interactions with other particles, as a means of exploiting reduction parallelism with little synchronization required. The resulting code is shown in Figure 15, where we must ensure that the array ETEMP is initialized to zero at the start of the program. The first $J$ loop may then be tiled as before, keeping it within the affinity region to maintain data locality and good load balance. Note that KSR Fortran does not support private arrays, and so the programmer is forced to use array expansion and indexing by process number. Thus for the first time the code is no longer a sequential code with annotations, as it now makes explicit reference to thread IDs and the number of threads being used.

The amount of work in the second loop is proportional to both the problem size $N$ and the number of threads $p$. It is possible to parallelize this loop, but it will only be worth doing so if the amount of work it contains is sufficient. A simplistic analysis suggests that it should be parallelized if $N(p-1)>c$ for some constant $c$. To parallelize this the loop order is inverted and a temporary scalar variable is introduced to minimize false sharing, as shown in Figure 16.

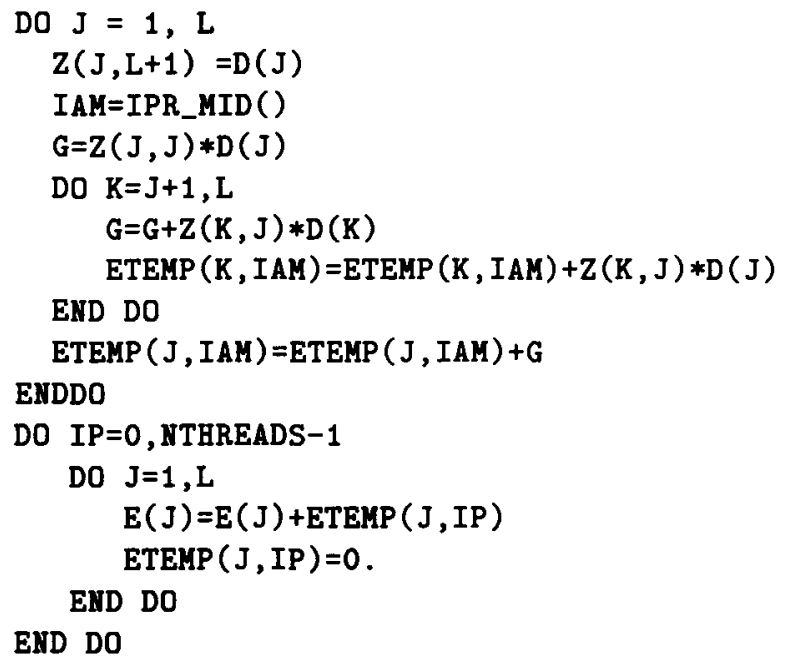

FIGURE 15 Fragment 1: alternative parallel version. 


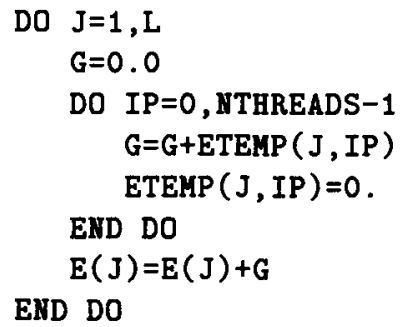

FIGURE 16 Fragment 1: parallelized reduction loop.

Note that the J loop is placed outermost to avoid the unnecessary synchronization which would result if it were innermost. This gives nonunit stride through ETEMP, but since each thread accesses only $N$ elements of ETEMP, and $N$ is much smaller than the number of words in the subcache, this should not result in any loss of performance due to subcache misses.

Since this loop nest has a rectangular iteration space and makes no reference to $Z$, there is no need for it to be within the affinity region. Therefore to minimize false sharing and tiling overheads the $\mathbf{J}$ loop is tiled using slice strategy. The constant $c$ is determined by experiment-a value of 1,000 is found to be satisfactory. Note that this parallelization strategy requires some $\mathbf{e x}^{-}$ tra memory (for the local copies of $\mathrm{E}$ ). The amount required is only $O(N p)$, compared to $O\left(N^{2}\right)$ for $\mathrm{Z}$, and is therefore not a significant added demand on memory.

Step 10: Alternative parallelization of Fragment 3.

Since the $J$ loop of Fragment 3 has a rectangular iteration space, the only reason for tiling it with mod strategy is to maintain data locality. However, since Fragment 3 is contained within a separate outer loop from Fragments 1 and 2, the resulting reduction in nonlocal memory accesses is not very significant. On the other hand, the use of mod strategy increases the overhead associated with tile generation, and causes a small amount of load imbalance, particularly when the length of the loop is small. Thus there is a trade-off situation, and experiments show that slice strategy, as described in Step 3, is slightly more efficient for this fragment, and so is readopted.

Step 11: Additional parallelism in Fragment 2.

In Step 4 the $J$ loop of Fragment 2 was split to give two loops, of which the first was tiled, but the sec- ond remained sequential. The decision not to tile the second loop was based on the amount of computation it contains. However, when the code is run using more than one thread, the cost of this loop increases, as it then produces a significant number of nonlocal memory accesses. If this loop is tiled (using mod strategy within the affinity region) this does not eliminate nonlocal accesses, but they are now distributed between threads, thus resulting in improved performance.

\section{EXPERIMENTAL RESULTS}

In this section we present the results of running various versions of TRED2 on the KSR-1. Results are given for three versions:

1. Compiler Version developed by the "compiler" in Section 4.

2. Programmer Version developed by the programmer in Section 5.

3. KAP Version obtained by preprocessing the original source code with KAP.

All versions were compiled with the $-\mathrm{r} 8$ flag to ensure that double precision real variables are handled as 64-bit floating point numbers. Without this flag, they are handled as 128-bit numbers, and floating point arithmetic is done in software). The KAP version was preprocessed with the flags - popt $=2$-autotile $=$ all (see $[10]$ ). The resulting programs were then run using the allocate_cells command to ensure one thread per cell execution and to avoid any time slicing with other processes in the machine. The initialization of the arrays and the printing of results are excluded from the timings. Table 2 shows the execution time in seconds for the three versions, for problem sizes $N=128,256,512$, 1,024 , and 2,048 respectively, with the exception that the KAP version was not run for the largest problem size, owing to the excessive time that would be required to do so.

Figures 17 to 19 present the same results for $N=128,512$, and 2,048 , in terms of temporal performance in solutions per second, which for TRED2 is simply the reciprocal of the execution time. The naive ideal performance is computed by multiplying the performance of the original sequential code by the number of processors; this is analogous to linear speedup on a speedup diagram. 
Table 2. Elapsed Times in Seconds for TRED2

\begin{tabular}{|c|c|c|c|c|c|c|}
\hline \multirow[b]{2}{*}{$N$} & \multirow[b]{2}{*}{ Version } & \multicolumn{5}{|c|}{ No. of Processors } \\
\hline & & 1 & 2 & 4 & 8 & 16 \\
\hline \multirow{3}{*}{128} & Compiler & 5.1 & 2.9 & 1.7 & 1.2 & 2.1 \\
\hline & Programmer & 4.2 & 2.6 & 1.6 & 1.2 & 1.1 \\
\hline & $\mathrm{KAP}$ & 3.6 & 2.7 & 2.2 & 1.9 & 1.9 \\
\hline \multirow{3}{*}{256} & Compiler & 43.2 & 22.7 & 12.3 & 7.2 & 5.2 \\
\hline & Programmer & 33.5 & 17.7 & 9.8 & 6.1 & 4.4 \\
\hline & $\mathrm{KAP}$ & 30.4 & 21.9 & 17.3 & 15.0 & 13.8 \\
\hline \multirow{3}{*}{512} & Compiler & 343 & 179 & 93.6 & 50.4 & 30.3 \\
\hline & Programmer & 264 & 137 & 71.7 & 39.0 & 23.3 \\
\hline & $\mathrm{KAP}$ & $24 \pi$ & 173 & 136 & 116 & 107 \\
\hline \multirow{4}{*}{1024} & Compiler & 2.772 & 1,408 & 728 & 379 & 211 \\
\hline & Programmer & 2.077 & 1.111 & 562 & 306 & 171 \\
\hline & $\mathrm{KAP}$ & 2,006 & 1,407 & 1.099 & $9+9$ & 874 \\
\hline & Compiler & 29.430 & 15,600 & 9.461 & 6.225 & 3.782 \\
\hline 2048 & Programmer & 22,870 & 11,420 & 5.033 & 2.523 & 1.417 \\
\hline
\end{tabular}

For $N=128$ performance gains in all parallel versions are poor. With such a small value of $N$ the granularity of the parallel computations is too fine for the KSR-1 to give significant performance improvement over the sequential version, and all the parallel versions are dominated by synchronization overheads. There is little to choose between Programmer and Compiler for this problem size, except on 16 processors, where Compiler takes a significant drop in performance. This is due to the effects of false sharing which become important when $N / p<16$, the number of words on a subpage. KAP is the fastest version on one processor, but it scales poorly, only just outperforming Compiler on 16 processors. Indeed, for all problem sizes up to 1,024, KAP performs very poorly, never achieving more than about two times the performance of the sequential code.

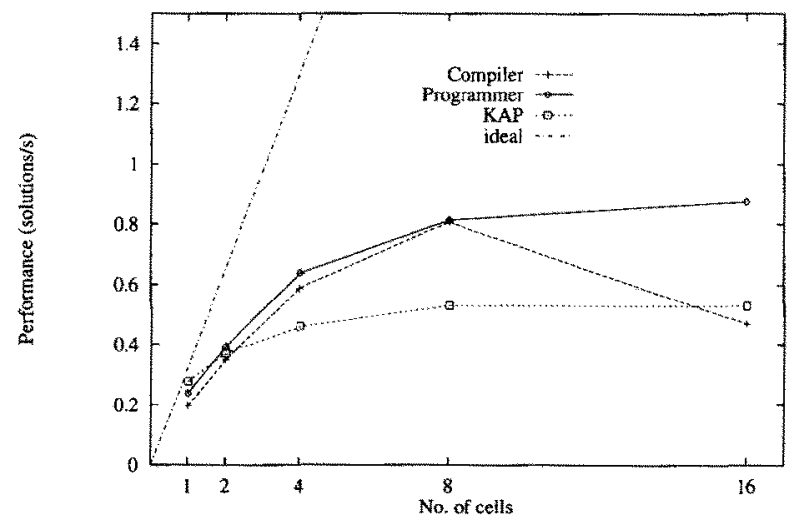

FIGURE 17 Temporal performance of TRED2, $N=$ 128.
With $N=256,512$, and 1,024, Programmer outperforms Compiler by 20 to $30 \%$ regardless of the number of processors. The scaling behavior of these two versions is very similar for these problem sizes, and steadily improves as the problem size increases.

For $N=2,048$, the memory requirement exceeds the memory available on a single cell. Thus for small numbers of processors, high numbers of page misses are generated. On this problem size, Programmer seales very well, with some superlinearity observed, whereas Compiler is again $30 \%$ slower on one processor, but over 2.5 times slower on 16 processors.

\subsection{Human Effort}

The work described covers a long period and was concurrent with other projects and commitments.

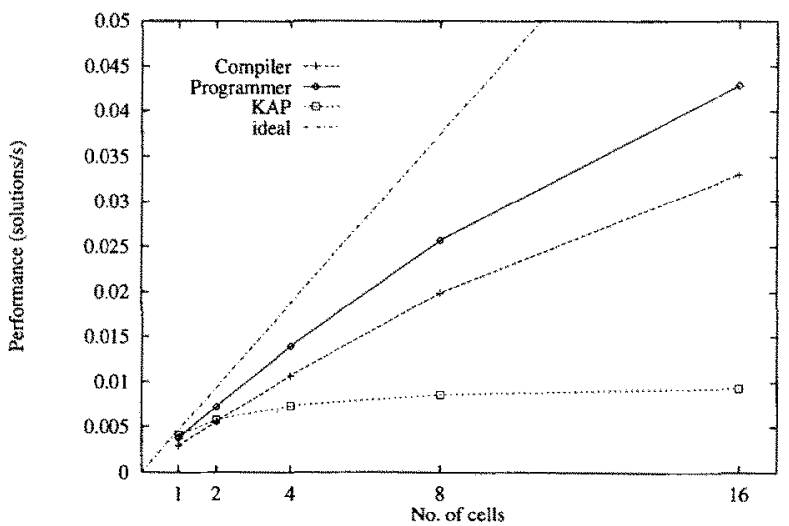

FIGURE 18 Temporal performance of TRED2. $N=$ 512. 


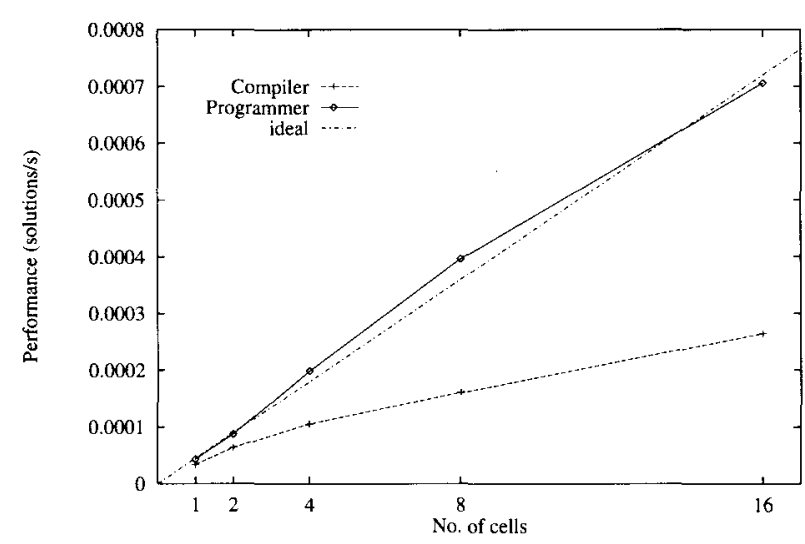

FIGURE 19 Temporal performance of TRED2. $N=$ 2,048 .

The programmer spent approximately eight working days optimizing the code over a 4-month period. In particular, Step 6 (obtaining a parallel version of Fragment 1 ) took about a day.

The compiler version took considerably more time. The strategy was based on previous work but applying it to a general Fortran program required 3 months research while the actual implementation on the KSR-1 took another 3 months. Ensuring that the synchronization points preserved program meaning (and did not deadlock) was the most time-consuming phase; the implementation of interleaving was a close second. The strategy has recently been implemented in Sage $++[3]$ taking 8 man-months.

\section{PERSPECTIVES}

\subsection{On the Compiler Method}

Many of the differences between the two approaches arise from the compiler and programmer employing distinctly different programming models. The compiler's model is based on the partition of data, whereas the programmer's model is essentially a shared memory approach. with emphasis on the partition of computation. Of course the programmer has to be aware that memory accesses are not uniform, but data layout is only considered when it is perceived that communication is contributing significantly to the overheads. On the other hand, the compiler approach is heavily data oriented, and the enforcement of the local-write rule results in code which is strongly reminiscent of a message-passing para- digm, even though there are no explicit messages. One effect of this difference is that the programmer has to make relatively few changes to the source code, while the compiler code is almost unrecognizable from the sequential version, and is also difficult to read. With the continuing poor performance of "black-box" style parallelizing compilers, it is becoming clear that it is necessary for the programmer to be able to understand and influence compiler decisions. Because the compiler's output code is so hard to interpret, the compiler's decisions would have to be communicated to the programmer via some interface other than simply the transformed code.

As is clear from the programmer's analysis of Fragment 3, the data-oriented approach is not always optimal. In this case the load imbalance in the affinity-based schedule employed by the compiler outweighs the communication costs in the programmer's version. Indeed, load balancing appears to be a weak area in the compiler strategy. While, in general, the programmer is able to design quite sophisticated schedules, the compiler is limited to interleaving, and even this seems messy and expensive to implement. The local-write rule employed by the compiler can be overrestrictive. Some parallel loops become sequentialized. and a sequential loop with data dependencies between iterations would require a lot of synchronization. Notice that the programmer does not have to worry about making local copies of remote data. as this is provided automatically by the ALLCACHE memory system.

The compiler's partitioning trade-off algorithm appears naive. It imposes an order on considerations which is program independent, takes no account of any machine parameters, and treats all significant regions as equally significant. Decisions are based on a scoring mechanism: a partition is chosen because it is optimal for the most significant regions, even though it could be disastrous for other regions, and therefore not the overall optimal choice. The compiler assumes that communication is more important than synchronization. For the KSR-1, which has relatively fast communication, but relatively slow synchronization, compared with other parallel architectures, this may not be a sound assumption. Having said all this, the compiler algorithm does do a good job on TRED2! The compiler does of course lack the advantage of profiling information to guide it, which the programmer usefully utilizes. Nevertheless, it would seem likely that the compiler would benefit, in the general case, from a more quantita- 
tive assessment of the overheads associated with each partitioning.

The compiler's programming model does have some marked benefits. The compiler is able to do transformations which would be very unpleasant to perform manually, and has no worries about the correctness of any transformations made. It appears considerably easier for the compiler to spot unnecessary synchronization and eliminate it. The compiler is also much better equipped to detect false sharing, and the local-write rule makes it relatively easy to avoid. For the programmer, false sharing can be quite difficult to detect. Note, however that the compiler does not take into account false sharing at the page (rather than subpage) level, which can result in excessive replication of data, though this problem is not encountered in TRED2.

Overall, the programmer's method results in faster code with far fewer changes to the original source. However, much of the method is reliant on intuition and experience, combined with the availability of detailed profiling information. The compiler is restricted to performance prediction rather than performance measurement. Its programming model appears unnecessarily restrictive, and it is clear that some architectural parameters should be taken into account in order to provide better performance prediction. Having said this for the test case we have used in this experiment, the compiler far outperforms the current commercially available compiler KAP, and comes quite close to the performance achieved by the programmer.

\subsection{On the Programmer Method}

One obvious difference between the two approaches is that the compiler approach applies a lot more analysis and transformations but is outperformed by the programmer. The major feature of the programmer method is the heavy use of profiling to guide program optimization. On the other hand it is somewhat ad hoc in nature. It seems that much of the knowledge of program and machine behavior is informal in nature and is not applied in a systematic step-wise manner so that it may be generalized for other programmers. However, this is a reasonable approach, if given a certain time constraint and a particular program to optimize, rather than programs in general. Clearly, modeling the KSR-1 as a single-level distributed memory machine gives encouraging results, but is too inaccurate to compete with the more intimate knowledge of the programmer.
The KSR programming model leaves much to be desired. It is considered that tiling innermost loops is not worthwhile due to tile overhead. However much of this tile overhead is due to unnecessary barrier synchronizations after parallel loops. This leads to the fusion of loops, trying to tile outer loops, and leaving inner parallel loops untiled. This unnecessary sequentialization also increases communication. All data referenced within a sequential loop will move to wherever the master thread is scheduled. An affinity-based approach keeps the data still and sequentializes by synchronization between processors. This is a KSR Fortran problem rather than a KSR-1 feature, yet is rarely considered worth tackling.

This compiler approach uses a static allocation of data based on the assumption that data movement due to write invalidation or false sharing is very expensive. This is certainly true in softwarebased SVM systems such as KOAN, but on the KSR-1 it seems that this is not always the case. In the third fragment of Figure 11 the programmer found that the overhead due to load imbalance using an affinity-based schedule is greater than that using a loop-based scheme.

Overall the main difference is that the programming approach is more effective per program in terms of performance and effort, but seems relatively ad hoc and, of course, has to be repeated for each application program. Initially there seems little that can be gained from one implementation to guide the next on a possibly different SVM machine. However, by carefully examining the techniques used, it seems possible that some may be incorporated into a compiler allowing the programmer to optimize at a higher level.

\subsection{On Current Differences and Future Developments}

At first glance, there may seem to be little commonality between the approaches used by optimizing compilers and programmers. This is particularly evident if one compares the resulting code. Perhaps the most significant difference is that programmers optimize in a cyclic manner (modify, run, repeat until satisfied) which is in stark contrast to the compiler's single pass approach. The programmer uses profiling information extensively, while the compiler does not use it at all. The approaches to program parallelization are notably different, with the compiler relying on loop distribution, while the programmer determines parallelism at the highest loop level, maintaining as much original structure as possible. 
Breaking a problem (multistatement imperfectly nested loop) into many simpler subproblems (multiple perfectly nested loops) is an attractive solution to the compiler, but for the programmer, the original code structure has meaning and it is easier to work with a few complex loop nests than multiple simpler ones. Nevertheless, there is definite common ground in that both approaches are concerned with reducing overheads, and both use a similar classification of overheads in order to guide decisions. Both programmer and compiler also use broadly similar techniques to reduce overheads, though the fact that each uses a different programming model tends to obscure this.

This experiment highlights a problem common to all compiler schemes which try to be applicable to a range of architectures however narrowly defined. The relative impact of any overhead will vary from machine to machine and should influence any compiler strategy. Rather than generating a new strategy for every machine type, what is needed is an explicit cost model based upon machine parameters, which will guide compilation. This model must incorporate those parameters that are relevant to the particular compilation model used and must guide rather than evaluate program transformations. Clearly, generating a possibly infinite number of programs and then checking it against a cost model is not practically feasible. Lnderstanding the important costs can only be gained from those using the machineprogrammers. By incorporating their knowledge into a compiler, some of the time-consuming process of parallelizing and mapping a program may be saved.

This experiment also exposes the essentially ad hoc nature of human parallel program development. Although we have shown that the programmer, through profiling and analysis of overhead sources, has a more or less systematic method of identifying problems in a parallel implementation, the solution of these problems still relies to a large extent on experience and intuition. There remains much potential to make this process more systematic, and hence more accessible to the nonexpert. The ability to work together with a compiler that is able to automate sophisticated transformations, give useful and intelligible feedback on its decisions, and possibly achieve some degree of performance prediction will be of significant benefit.

It is clear that the role of optimizing compilers in parallel scientific computing is as a tool to assist programmers, rather than as a replacement for them. If the compiler is to be an effective tool, then it will have to fit into the programmer's cyclic de- velopment method-this has a number of implications for the compiler. Firstly, the compiler must be able to follow directives placed in the program as well as perform optimizations automatically. Programmers will alwavs have domainspecific knowledge unavailable to the compiler and must therefore be allowed to override any compiler decision. Naturally, this implies that the compiler must report its decisions and the reasons why it made them in a manner that is understandable to the programmer. This information will allow the programmer to provide appropriate information/directives leading to a better implementation without requiring the programmer to completely restructure the code. Ideally, optimizing the program would consist of a number of iterations where different directives are inserted, rather than rewriting sections of the program. If we were to extend this idea, programmers would be encouraged to write their program in languages such as High Performance Fortran (HPF), Fortran90, or even higher level ones. This will aid portability and maintainability, as only the directives should have to change.

To facilitate this, optimizing compilers will have to be more open; each stage (parallelizing, mapping, etc.) must be able to take information from previous stages or from directives. They will also have to provide sensible implementations in the presence of partial direction. If, for example, the programmer specifies how one section of code is to be mapped, then the mapping of the remaining program sections should accommodate this information. This is essential if compilers are to evolve beyond concentrating on local loop analysis to global program-wide knowledge.

A prototype of the compiler strategy described in the article is now implemented. The compiler is to be extended in the light of this experiment. Each of the compiler phases will be broken into units where important decisions are based on input values rather than hard-coded internal algorithms. These values may be generated by earlier phases or directly from directives. This allows variable control by the programmer and, because of modularity, simplifies targeting the compiler for different platforms and incremental addition of functionality.

Since the compiler can at best contain a relatively simple model of machine performance, programmers will want to continue to use profile information to guide optimization even if they are working together with a sophisticated compiler. Thus for maximum benefit a compiler should be part of an integrated development environment 
that supports editing, interactive compilation, running programs, performance monitoring. and presentation of profiling information. The challenge is to define a directive system which allows higher-level control than, say, enforcing a particular loop transformation. This will include control of execution model (SPMD, fork/join, multithreading) and strategy where the relative cost of overheads can be defined (i.e., load balance. communication, synchronization, etc.). Giving the "right" information back to the user is essential and this must be defined by the user. Experience within our group has shown that different users have different requirements and that interfaces must allow some customization. The programmer will have control on the level of detail in compiler feedback and will be able to view the program in a number of ways, e.g., by significant regions, variable name, synchronization point, etc. The FORGE system is an early example of such a system $[1]$.

\section{CONCLUSION}

For the case of TRED2, for which it is possible to completely statically analyze data accesses, but is otherwise nontrivial to optimize for parallel execution, the compiler strategy described in Section 3 is shown to give almost comparable performance to an expert programmer, and to outperform the commerically available parallelizing compiler, $\mathrm{KAP}$, by a significant margin.

Through the detailed description of the application of programmer and compiler strategies to TRED2, both differences and common ground between the two strategies have been highlighted. Strengths and weaknesses of the strategies have been identified, giving insight into how the two approaches should be integrated in a unified program development environment.

Future experiments on different programs and architectures will aid the development of the compiler and its user interface. This system will allow the programmer to concentrate on those areas where the compiler is poor and try complex strategies which would be too costly to try by hand. In this way it is hoped that a more useful partnership between programmer and compiler will be achieved, resulting in a systematic combined strategy allowing rapid development of efficient parallel programs.

\section{REFERENCES}

[1] A. P. R., Forge 90: Automatic Parallelizer for High Performance Fortran 77 on Distributed Memory System User's Guide. Placerville. CA: Applied Parallel Research, 1993.

[2] F. Bodin and M. O'Boyle, A Compiler Strategy for SVM, in B. Szymanski and B. Sinharoy. Eds., Languages, Compilers and Run-Time Systems for Scalable Computers, Kluwer Academic Publishers, May 1995, pp. 5?-68.

[3] F. Bodin, P. Beckman, D. Gannon, and J. G. S. Srinivas, "Sage ++ : A Class Library for Building Fortran and $\mathrm{C}++$ Restructuring Tools," presented at the Second Object-Oriented Numerics Conference, Oregon, April 1994.

[4] H. Burkhart and R. Millen. "Performance-measurement tools in a multiprocessor environment." IEEE Trans. Comput., vol. 38. pp. 725-737. 1989.

[5] M. E. Crovella and T. J. LeBlanc, "The search for lost cycles: a new approach to parallel program performance evaluation," Lniversity of Rochester, Department of Computer Science. Tech. Rep. 479. December 1993.

[6] G. K. Egan. G. D. Riley, and J. M. Bull 'Parallelisation of the SDEM distinct element stress analysis code on the KSR-1, " in Proc. ACV International Conference on Supercomputing. pp. 85-92. 1994

[7] C. Falcó Korn, J. M. Bull. G. D. Riley, and P. K. Stansby, "Parallelisation of a three-dimensional shallow water estuary model on the KSR-1," Sci. Prog. vol 4, no. 3, pp. 155-170. 1995.

[8] M. Gupta, "Automatic data partitioning on distributed memory multicomputers." Lniversity of Illinois at Urbana-Champaign, Centre for Reliable and High Performance Computing. Tech. Rep. UILL-EXG-92-2237, CRHC-92-19, September 1992.

[9] W. Kelly and W. Pugh, "Generating schedules and code within a unified reordering transformation framework," University of Maryland Tech. Rep. UMIACS-TR-92-126, August 1992.

[10] K. S. R., KSR Fortran Programming, Waltham. MA: Kendall Square Research, 1992.

[11] J. Li and M. Chen, "Index domain alignment: minimising cost of cross-referencing between distributed arrays," in IEEE Proc. Third Symposium on the Frontiers of Massively Parallel Computation, pp. 424-433, 1990.

[12] L.-C. Lu, "A unified framework for systematic loop transformations," presented at the 3rd ACM Symposium on the Principles and Practice of Parallel Programming, 1991.

[13] M. F. P. O'Boyle, "Program and data transformation for efficient execution on distributed memory architectures," University of Manchester, Tech. Rep. 93-1-6, January 1993. 
[14] M. F. P. O'Boyle, " $\Lambda$ data partitioning algorithm for distributed memory compilation." in Proc. of PARLE 94. New York: Springer Verlag; 1994.

[15] M. F. P. O'Boyle, "Hierarchical locality optimisations for NUMAs." in Proc. EuroMicro PDP, Sanremo, pp. 106-114. 1995.

[16] D. A. Padua and M. J. Wolfe, "Advanced compiler optimizations for supercomputers," Commun. $A C M$ vol. 29, pp. 1184-1201, 1986.

[17] W. Pugh, 'Uniform techniques for loop optimisation," presented at the International Conference on Supercomputing, 1991.

[18] M. Wolfe, "Optimising supercompilers for supercomputers," in C. Jesshope and D. Klappholz, Eds., Research Monographs in Parallel and Distributed Computing. London: Pitman. 1989.

$19]$ M. E. Wolf and M. Lam. "An algorithmic approach to compound loop transformations," in Advances in Languages and Compilers for Parallel Processing. A. Nicolau. D. Gelernter, T. Gross. and D. Padua, Eds.. London. pp. 243-259. 1991

\section{Appendix 1. TRED2 listing}

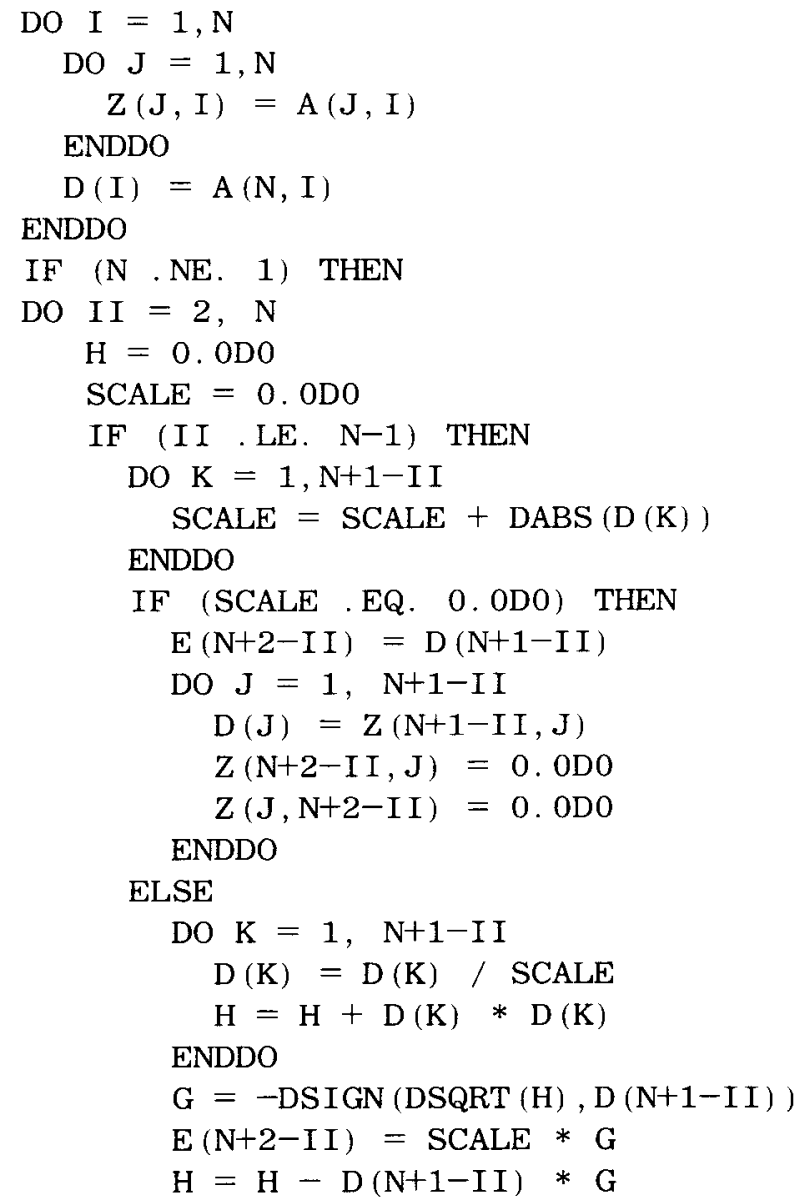

b:

a:

c:

$$
\begin{aligned}
& \text { DO } \mathbf{J}=1, \mathrm{~N}+1-\mathrm{II} \\
& \text { DO } \mathrm{K}=\mathrm{J}, \mathrm{N}+1-\mathrm{II} \\
& \mathrm{Z}(\mathrm{K}, \mathrm{J})=\mathrm{Z}(\mathrm{K}, \mathrm{J})-\mathrm{D}(\mathrm{J}) \\
& \text { ENDDO } \\
& \mathrm{D}(\mathrm{J})=\mathrm{Z}(\mathrm{N}+1-\mathrm{II}, \mathrm{J}) \\
& \mathrm{Z}(\mathrm{N}+2-\mathrm{II}, \mathrm{J})=0.0 \mathrm{D} 0 \\
& \text { ENDDO } \\
& \text { ENDIF } \\
& \text { ELSE } \\
& E(N+2-I I)=D(N+1-I I) \\
& \text { DO } \mathrm{J}=1, \mathrm{~N}+1-\mathrm{I} \mathrm{I} \\
& \mathrm{D}(\mathrm{J})=\mathrm{Z}(\mathrm{N}+\mathrm{1}-\mathrm{II}, \mathrm{J}) \\
& \mathrm{Z}(\mathrm{N}+2-\mathrm{II}, \mathrm{J})=0.0 \mathrm{D0} \\
& \mathrm{Z}(\mathrm{J}, \mathrm{N}+2-\mathrm{II})=0.0 \mathrm{DO} \\
& \text { ENDDO }
\end{aligned}
$$$$
\text { NDDO }
$$$$
* \mathrm{E}(\mathrm{K})-\mathrm{E}(\mathrm{J}) * \mathrm{D}(\mathrm{K})
$$ 
d:

e:
$G=G+Z(K, I)$

$$
\text { * } \mathrm{Z}(\mathrm{K}, \mathrm{J})
$$

ENDDO

DO $\mathrm{K}=1, \quad \mathrm{I}-1$

$\mathrm{Z}(\mathrm{K}, \mathrm{J})=\mathrm{Z}(\mathrm{K}, \mathrm{J})-\mathrm{G}$

ENDDO

ENDDO

ENDIF

DO $\mathrm{K}=1, \mathrm{I}-1$
$\mathrm{Z}(\mathrm{K}, \mathrm{I})=0.0 \mathrm{DO}$ ENDDO

ENDDO

ENDIF

DO $\mathrm{I}=1, \mathrm{~N}$

$\mathrm{D}(\mathrm{I})=\mathrm{Z}(\mathrm{N}, \mathrm{I})$

$\mathrm{Z}(\mathrm{N}, \mathrm{I})=0.0 \mathrm{DO}$

ENDDO

$\mathrm{Z}(\mathrm{N}, \mathrm{N})=1.0 \mathrm{DO}$

$\mathrm{E}(1)=0.0 \mathrm{D} 0$ 

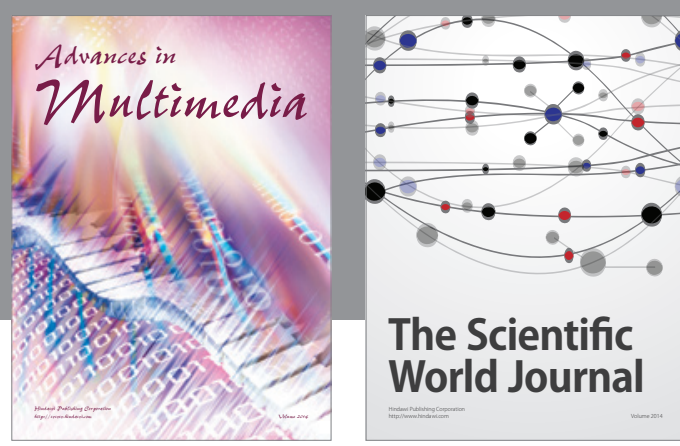

The Scientific World Journal
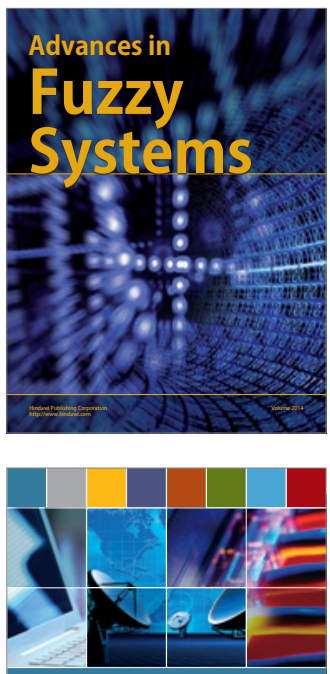

Computer Networks and Communications
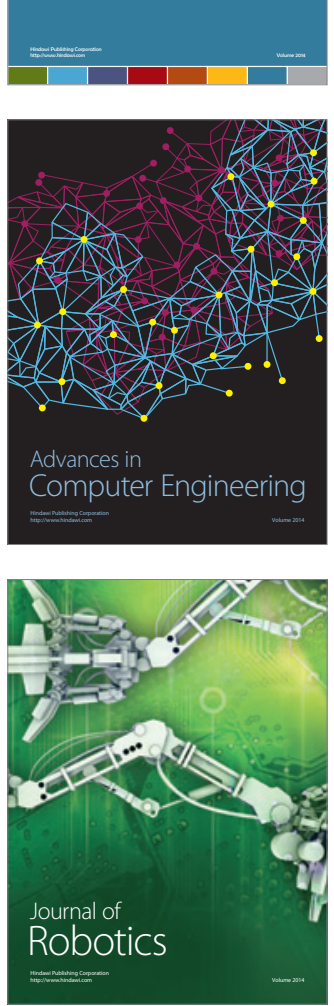
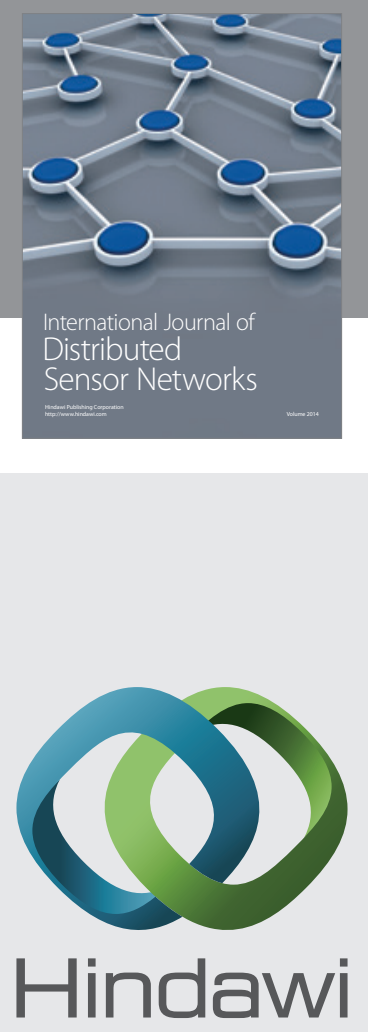

Submit your manuscripts at

http://www.hindawi.com
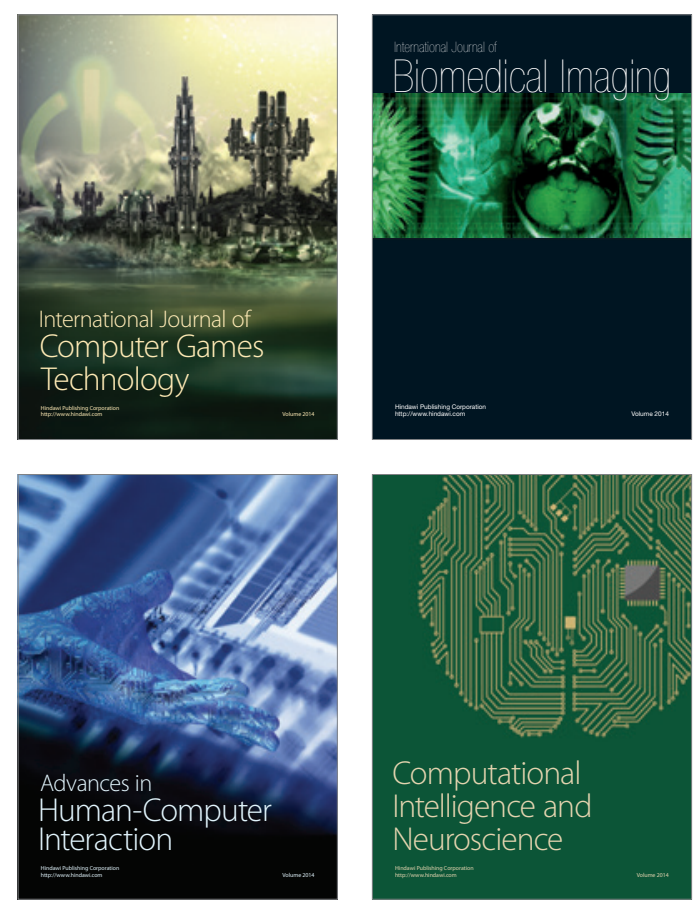
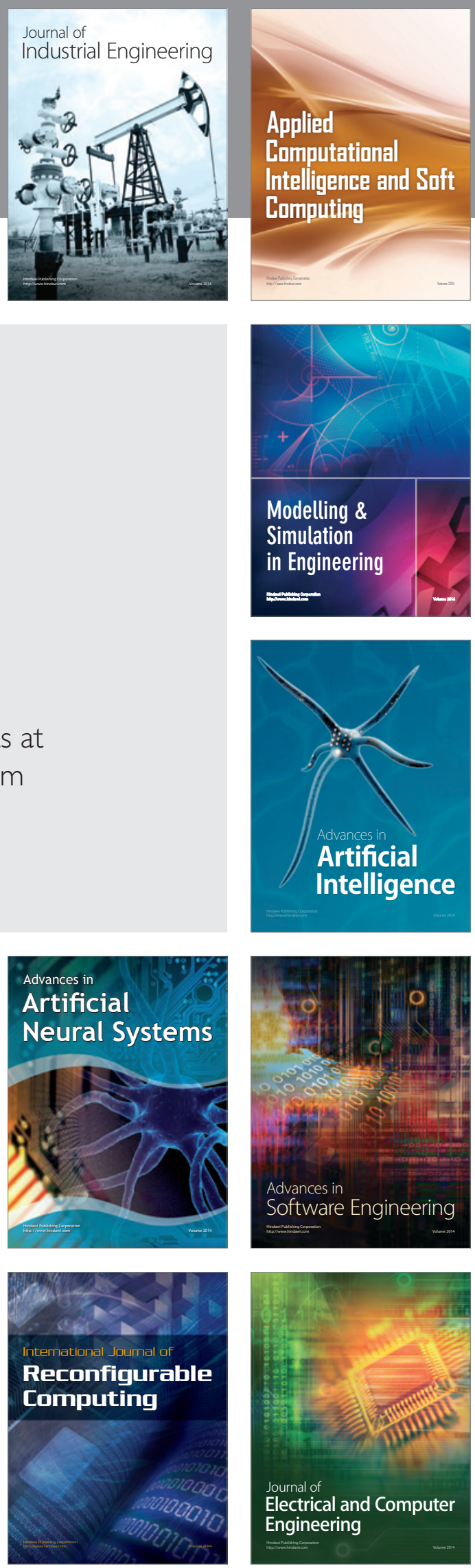